\title{
Dynamic simulation of dispersed gas-liquid two-phase flow using a discrete bubble model
}

\author{
E. Delnoij, * F. A. Lammers, J. A. M. Kuipers and W. P. M. van Swaaij \\ Department of Chemical Engineering. Twente University, P.O. Box 217.7500 AE Enschede. \\ The Netherlands
}

(Received 4 September 1996; in revised form 3 December 1996; accepted 9 December 1996)

\begin{abstract}
In this paper a detailed hydrodynamic model for gas-liquid two-phase flow will be presented. The model is based on a mixed Eulerian-Lagrangian approach and describes the time-dependent two-dimensional motion of small, spherical gas bubbles in a bubble column operating in the homogeneous regime. The motion of these bubbles is calculated from a force balance for each individual bubble, accounting for all relevant forces acting on them. Contributions from liquid-phase pressure gradient, drag, virtual mass, liquid-phase vorticity and gravity are considered, whereas direct bubble-bubble interactions are accounted for via an interaction model resembling the collision model developed by Hoomans et al. (1996) to model gasfluidized beds. The liquid-phase hydrodynamics are described using the volume-averaged, unsteady, Navier-Stokes equations. A preliminary model validation has been performed by comparing the computational results with experimental observations published previously in literature by various authors. The model is shown to predict correctly the motion of a bubble plume in a pseudo-two-dimensional bubble column operated at different superficial gas velocities, provided that a detailed description of the bubble dynamics is incorporated in the model. The effect of bubble column aspect ratio on the hydrodynamic behaviour of the column has also been investigated. Our model predicts the effect of aspect ratio on the flow structure in the bubble column. The importance of the various forces acting on the bubbles will also be discussed and it will be shown that the added mass force and the lift force cannot be neglected in bubble column simulation. Finally, the model has been used to study the start-up behaviour of a two-dimensional bubble column. It will be shown that the history of the gas-liquid two-phase flow significantly affects the flow structure ultimately obtained in a bubble column. This finding has, to our knowledge, not been reported before in literature. C. 1997 Elsevier Science Ltd. All rights reserved
\end{abstract}

Keywords: Bubble columns; two-phase flow; hydrodynamics.

\section{INTRODUCTION}

Bubble column reactors are frequently employed in the biological, chemical and petrochemical industry. Compared to other multiphase reactors, such as packed towers and trickle bed reactors, bubble columns offer some distinct advantages, among which its simple construction and excellent heat transfer characteristics can be mentioned. The biggest disadvantage, on the other hand, is the limited knowledge about the fluid dynamics of these gas-liquid twophase systems, despite the significant research efforts which have been made both in academic and industrial research laboratories. Modelling the fluid $\mathrm{dy}$ namics of gas-liquid bubble columns is therefore still a challenging problem.

*Corresponding author: E-mail: e.delnoij@ct.utwente.nl.
There are two well-known approaches to model gas-liquid two-phase flows. One approach is based on the mixture formulation in which effective (transport) properties are used to describe these two-phase flows. This approach is relatively simple to use, but cannot account for any interaction (i.e. slip, mass and heat transfer) between the phases. In the second approach both phases are treated separately where transfer of mass, heat and momentum between the phases can be accounted for. Within the context of this approach one can distinguish Eulerian/Eulerian (or two fluid) models which are based on the concept of interpenetrating continua, and Eulerian/Lagrangian models which adopt a continuum description for the liquid phase and additionally track each individual bubble using the Newtonian equations of motion.

In recent years a number of authors (Torvik and Svendsen, 1990; Ranade, 1992; Grienberger and 
Hofman, 1992; Hjertager and Morud, 1995; and Sokolichin and Eigenberger, 1994) have published interesting results obtained with two fluid gas-liquid models. Among others Sokolichin and Eigenberger have contributed to the development of this type of model. Their calculations clearly demonstrate the time-dependent behaviour of a gas-liquid bubble column, and thus stress the importance of dynamic models. Long time averaging of their instantaneous velocity distributions leads to the familiar, experimentally observed, liquid velocity profile with liquid upflow in the column centre and liquid downflow near the walls of the column.

Due to advances in computer technology Eulerian/Lagrangian models for gas-liquid bubble columns have received increased attention in recent years. Pioneering work in this field was conducted by Trapp and Mortensen (1993), Lapin and Lübbert (1994) and Devanathan et al. (1995). The models developed differ in their description of the bubble dynamics, and in the representation of the coupling between the phases. In their recent paper Lapin and Lübbert developed a two-dimensional Eulerian/Lagrangian model of a bubble column using a simple description of the bubble dynamics. Coupling between the gas and the liquid phase was achieved through the effective density of the mixture. No exchange of momentum between the phases was incorporated in their model.

In the present paper we will present a detailed Eulerian/Lagrangian model for a gas-liquid bubble column operating in the homogeneous regime. The liquid phase will be described using the well-known volume averaged Navier-Stokes equations whereas the dispersed phase will be described by the equations of motion for each individual bubble. The exchange of momentum between the gas and the liquid phase will also be accounted for. In addition, bubble dynamics will be described in detail, incorporating all relevant forces acting on a bubble rising in a liquid. In order to prevent, physically impossible, bubble-bubble overlap, direct bubble-bubble interactions will be described using a collision model. Finally a preliminary experimental validation of the model will be reported using experimental results obtained from the literature.

\section{BUBBLE DYNAMICS}

Gas-liquid bubble columns can be operated in a number of different regimes. The flow regime encountered in the column depends on one hand on the superficial gas velocity and the physical properties of the phases, and on the other hand, on the aspect ratio of the column. One can distinguish the homogeneous or dispersed bubble regime, the heterogeneous or churn turbulent regime and the slug flow regime (Deckwer and Schumpe, 1993). The homogeneous flow regime is characterised by relatively low gas velocities and small, spherical bubbles. In a bubble column operating in the heterogeneous regime, i.e. at intermediate gas velocities, a large number of different bubbles with varying size and shape can be observed. Bubble shapes range from spherical to spherical cap. Slug flow occurs at high gas velocities in small diameter bubble columns.

As stated in the Introduction, the model developed applies to the homogeneous or dispersed bubble regime. This implies that the bubbles can be considered as spherical, which simplifies the calculation of the force exerted by the liquid on the bubbles significantly. We will assume that this force $\mathbf{F}_{\text {total }}$ acting on a non-deformable spherical bubble rising in an unsteady, and non-uniform liquid flow is composed of separate and uncoupled contributions from pressure gradient, drag, virtual or added mass, vorticity and gravity (Auton, 1983):

$$
\mathbf{F}_{\text {total }}=\mathbf{F}_{P}+\mathbf{F}_{D}+\mathbf{F}_{V M}+\mathbf{F}_{L}+\mathbf{F}_{G} .
$$

The acceleration of a bubble can then be calculated from a force balance over that bubble:

$$
m_{b} \frac{\mathrm{d} \mathbf{v}}{\mathrm{d} t}=\mathbf{F}_{\text {total }}
$$

where $m_{b}$ represents the mass of a bubble. Once the bubble acceleration is know, the new bubble velocity can be calculated using a simple, explicit integration formula:

$$
\mathbf{v}^{n+1}=v^{n}+\left(\frac{\mathrm{d} v}{\mathrm{~d} t}\right)^{n+1} \mathrm{D} T
$$

which states that the new bubble velocity equals the sum of the bubble velocity at the previous time level and the most recently obtained acceleration of the bubble multiplied by the time step DT. The calculation of the new bubble positions, from their new velocities and previous time level positions, requires to account for possible direct bubble-bubble interaction (collisions) as will be described in Section 2.5. First the various forces acting on a bubble will be discussed in more detail.

\subsection{Gravity and far-field pressure contribution}

The force acting on a bubble due to the pressure gradient in the liquid incorporates contributions from the Archimedes displacement force, inertial forces and viscous strain in the liquid. The sum of liquid-phase pressure gradient and gravity equals:

$$
\mathbf{F}_{G}+\mathbf{F}_{\boldsymbol{P}}=\rho_{g} V_{b} \mathbf{g}-V_{b} \nabla \mathbf{P}
$$

\subsection{Drag force}

A bubble moving with a constant velocity through a uniform liquid flow field experiences a drag force, which consists of a form drag and a friction drag exerted by the liquid on the moving bubble. The drag force acting on a suspended sphere is given by Odar (1964), and is found to be proportional to the relative velocity between the phases as follows:

$$
\mathbf{F}_{D}=-\frac{1}{2} C_{D} \rho_{l} \pi \mathrm{R}_{b}^{2}|\mathbf{v}-\mathbf{u}|(\mathbf{v}-\mathbf{u}) .
$$


The drag coefficient $C_{D}$ depends on the flow regime and the liquid properties. For rigid spheres the drag coefficient is usually approximated by the standard drag curve (Clift et al., 1978):

$$
C_{b}=\left\{\begin{array}{l}
R_{e}<1000 \Rightarrow \frac{24}{R_{e}}\left(1+0.15 R e^{0.687}\right) \\
R e \geqslant 1000 \Rightarrow 0.44 .
\end{array}\right.
$$

However, because of internal gas circulation and deformation, bubbles do not necessarily behave as rigid spheres; their drag coefficient can therefore differ from that predicted by the standard drag curve. According to Clift $c^{\prime} t$ l., the drag on bubbles in pure water is less than the drag predicted by the standard drag curve. In contaminated systems, on the other hand, the surfactants tend to collect at the rear of the bubble whereby the slip along the surface of the bubble is reduced. In contaminated systems, therefore, bubbles behave more like rigid particles (Auton, 1983). As tap water is used in most experiments reported in the literature, it is decided to use the standard drag curve equation as an estimate for the drag coefficient of a spherical bubble.

\subsection{The lift force}

Bubbles rising in a non-uniform liquid flow field, experience a lift force due to vorticity or shear in the liquid flow field. Auton (1983) calculated the lift force exerted by an inviscid liquid on a bubble in a vertical. linear shear flow' represented by:

$$
u_{y}=\left(\kappa x+u_{\text {rise }}\right)
$$

He derived that the lift force depends on the vector product of the slip velocity and the curl of the liquid velocity, resulting in a lift force that acts in a direction perpendicular to both the direction of the slip velocity and the direction of the curl of the liquid velocity field. If the bubble velocity exceeds the liquid velocity the lift force is directed towards a region with a lower liquid velocity. If the bubble moves with a lower velocity the lift force is directed towards the high velocity region:

$$
\begin{aligned}
\mathbf{F}_{L} & =-C_{L} \rho_{l} V_{h}(\mathbf{v}-\mathbf{u}) \times \Omega \\
\Omega & =\nabla \times \mathbf{u} .
\end{aligned}
$$

Expression (8) for the lift force is valid under the assumption of local homogeneity of the flow, or:

$$
\frac{R_{h} K}{u_{\text {rise }}} \ll 1 .
$$

This assumption is satisfied throughout the homogeneous regime where both bubble radius and liquid velocity gradients are rather small; therefore, Auton's lift force equation has been implemented in our model. The value of the lift coefficient has been calculated by Auton (1983) and was found to be equal to 0.53 .

\subsection{The virtual mass force}

The total force exerted by the liquid on the bubble is in part independent of changes in the slip velocity between the phases and in part dependent on changes in the slip velocity. This latter part can be seen as a resistance to acceleration. This resistance to acceleration is due to an "added mass" of liquid that has to be accelerated when a bubble accelerates. According to Auton (1983) this added or virtual mass force can be modelled using eq. (10):

$$
\begin{aligned}
\mathbf{F}_{v, M} & =-\left(\frac{\mathrm{D} \mathbf{I}}{\mathrm{D} t}+\mathbf{I} \cdot \nabla \mathbf{u}\right) \\
\mathbf{I} & =C_{{ }^{\prime} M} \rho_{l} V_{b}(\mathbf{v}-\mathbf{u}) .
\end{aligned}
$$

The material derivative in this equation for the virtual mass force should be the derivative pertaining to the gas bubble. Van Wijngaarden (1976) argued that the influence of neighbouring bubbles on the virtual mass coefficient of the bubble under consideration can be expressed in terms of the average void fraction in the vicinity of that bubble as follows:

$$
C_{M}^{\prime}=C_{M M}[1+2.78(1-i)] .
$$

For all computations reported in this paper the value for the virtual mass coefficient $C_{V M}$ has been taken to equal 0.5 .

\subsection{Direct bubble - bubble interaction}

When two bubbles approach each other. the liquid between the bubbles will resist this relative motion. Due to this motion the pressure in the liquid film between the bubbles increases and as a consequence the liquid is forced to move out of this film. The final outcome of this process depends on the magnitude of the pressure in the liquid film between these iwo bubbles. If the pressure is high enough to stop the relative motion of the bubbles, the bubbles will bounce; otherwise bubble coalescence will result. Duineveld (1994) showed that the Weber number based on either the rise velocity of the bubbles or the approach velocity of the bubbles determines whether two bubbles will coalesce, bounce and then coalesce or bounce and separate. He determined a minimum bubble size for bouncing and separation of air bubbles in pure water of $1.72 \mathrm{~mm}$. Assuming a bubble size exceeding this minimum bubble size, but small enough to assume a spherical bubble shape. a collision model can be used to describe the bouncing and separation of two bubbles.

The processing of a sequence of collisions is hased on the method developed by Hoomans et al. (1996). In this method a constant time step DT is used to account for the forces acting on a bubble. Within this time step, the velocity of the bubbles is assumed to change only due to binary collisions between bubbles; a sequence of collisions is then processed one collision at a time.

In this computation a collision between two bubbles is supposed to occur when the distance between these two bubbles equals the sum of the two 
radii of the respective bubbles plus an added volume equivalent to the volume occupied by the virtual mass of liquid:

$$
\left|\mathbf{r}_{a}-\mathbf{r}_{b}\right|=\left(R_{a}+R_{b}\right) \sqrt[3]{1+C_{V M}} .
$$

In order to process a sequence of collisions, the time to collision for each bubble with another bubble or one of the containing walls has to be determined. This time step $t_{a b}$ can be calculated from the initial position of the bubbles and their velocities. This yields a quadratic equation in $t_{a b}$, in which the smallest positive root corresponds to the collision time: updated using this time interval and the bubble velocities [obtained from eq. (3)] as follows:

$$
\mathbf{r}_{a}\left(t+t_{a b}\right)=\mathbf{r}_{a}(t)+\mathbf{v}_{a} t_{a b} .
$$

However, within this time interval $t_{a b}$ there are at least two bubbles that bounce. Their velocities change due to this collision as depicted in Fig. 1. In the first step the velocities of the individual bubbles are split into two components, one along the line connecting the centres of mass of both bubbles and one perpendicular to that line. Three simplifying assumptions are made to calculate the new velocities; the collisions are

$t_{a b}=\frac{-b_{a b}-\sqrt{b_{a b}^{2}-\left|\mathbf{v}_{a}-\mathbf{v}_{b}\right|^{2}}\left(\left|\mathbf{r}_{a}-\mathbf{r}_{b}\right|^{2}-\left(R_{a}+R_{b}\right)^{2}\left(1+C_{V M}\right)^{2 / 3}\right)}{\left|\mathbf{v}_{a}-\mathbf{v}_{b}\right|^{2}}$

where

$$
b_{a b}=\left(r_{a x}-r_{b x}\right)\left(v_{a x}-v_{b x}\right)+\left(r_{a y}-r_{b y}\right)\left(v_{a y}-v_{b y}\right) .
$$

As the time interval to the next collision has been calculated for each bubble, the smallest collision time of all bubble pairs contained in the column can be determined. The bubble positions are subsequently

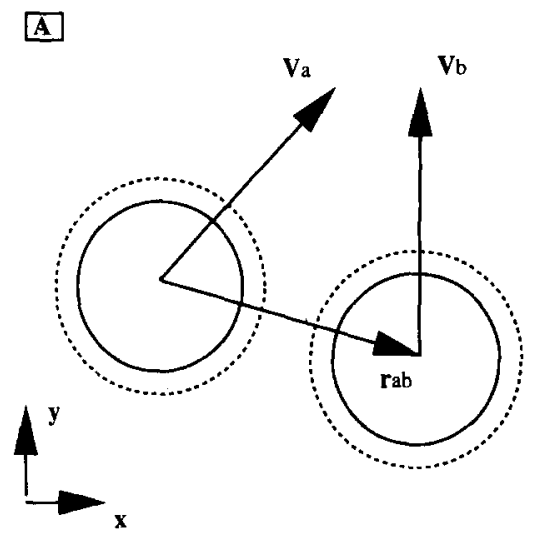

[C]

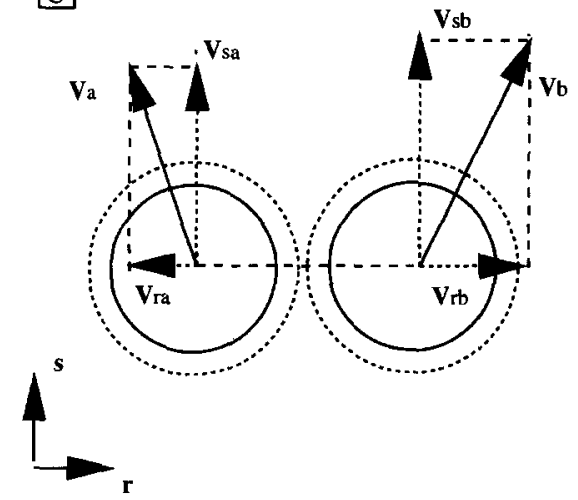

instantaneous, completely elastic and the rotation of the bubbles remains unaffected by the collision. Therefore, the velocity component perpendicular to the centreline does not change, whereas the velocity component along the centreline can be computed from:

$$
\left(v_{r a}\right)_{\mathrm{after} \text { collision }}=2 \frac{m_{a} v_{r a}+m_{b} v_{r b}}{m_{a}+m_{b}}-v_{r a}
$$

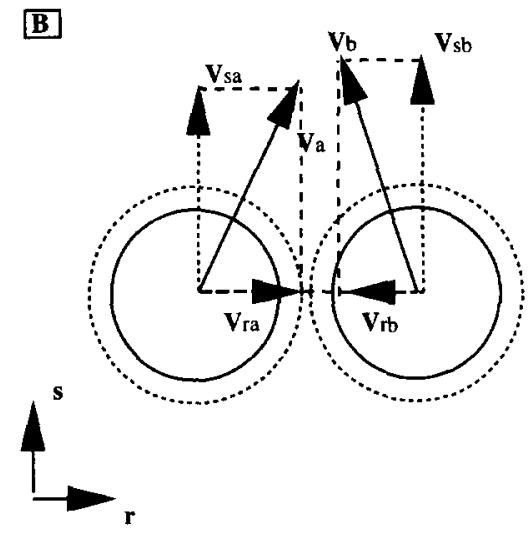

D

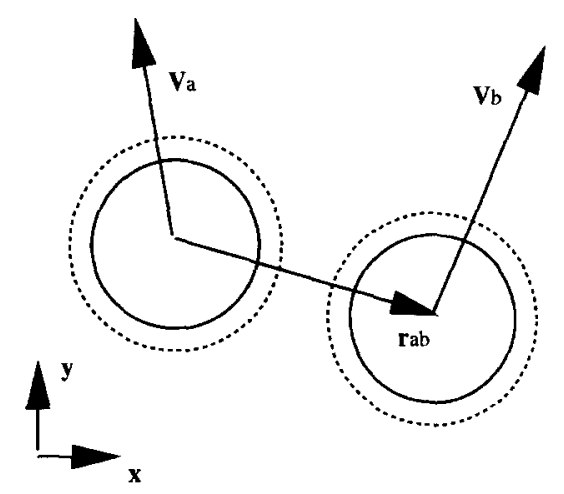

Fig. 1. New velocities of two bouncing bubbles are calculated using a collision sub model: (A) bubbles in Cartesian grid; (B) colliding bubbles in coordinate system used to describe the collisions; (C) new velocities from collision dynamics; (D) new Cartesian velocities. 
The final step consists of calculating the Cartesian velocities of both bubbles from the velocity components used in the collision coordinate system. The interested reader is referred to the paper of Hoomans et al. for additional details.

\section{LIQUID-PHASE HYDRODYNAMICS}

\subsection{Governing equations}

The liquid-phase hydrodynamics are described using the volume-averaged mass and momentum conservation equations:

$$
\begin{gathered}
\frac{\partial\left(\varepsilon_{l} \rho_{l}\right)}{\partial t}+\nabla \cdot \varepsilon_{l} \rho_{l} \mathbf{u}=\mathbf{0} \\
\frac{\partial\left(\varepsilon_{l} \rho_{l} \mathbf{u}\right)}{\partial t}+\nabla \cdot \varepsilon_{l} \rho_{l} \mathbf{u u}=-\varepsilon_{l} \nabla \mathbf{P}-\nabla \cdot \varepsilon_{l} \tau_{l}+\varepsilon_{l} \rho_{l} \mathbf{g}+\Phi .
\end{gathered}
$$

The spatial resolution with which the liquid velocity field is resolved is small compared to the size of the bubbles; the liquid-bubble interaction is therefore superimposed on the liquid velocity field. This liquid-bubble interaction is modelled using a source term $\Phi$ which accounts for the momentum exchange between the bubbles and the liquid.

In the present study two-dimensional, isothermal motion of both the gas and the liquid phase is assumed. The basic variables that have to be calculated from the model are pressure, liquid velocity and the velocity and position of each individual bubble. All other variables in the balance equations must be specified in terms of these basic variables.

\subsection{Constitutive equations}

The liquid-phase viscous stress tensor $\tau_{l}$ is modelled assuming general Newtonian behaviour of the liquid:

$\tau_{l}=-\cdots\left[\left(\lambda_{l}-\frac{2}{3} \mu_{l}\right)(\nabla \cdot \mathbf{u}) E+\mu_{1}\left((\nabla \mathbf{u})+(\nabla \mathbf{u})^{\mathrm{T}}\right)\right]$

The bulk viscosity $i_{1}$ is set to zero in all simulations presented in this paper. In most simulations water is used as the liquid phase which corresponds to a shear viscosity of $1.0 \times 10^{-3} \mathrm{~kg} \mathrm{~m}^{-1} \mathrm{~s}^{-1}$.

\subsection{Coupling between bubbles and liquid and vice versa}

In our model the coupling between the gas and the liquid phase appears through the liquid volume fraction $a_{1}$ and the source term $\Phi$ in the liquid-phase momentum equation which represents momentum exchange between the gas phase and the liquid phase. As the system of partial differential equations governing the liquid-phase hydrodynamics is solved using a finite differencing technique this source term has to be calculated in accordance with the number of bubbles present in a differential volume element and the individual velocities of these bubbles. This provides the momentum transfer from the bubbles to the liquid; the liquid on the other hand, exerts a force on the bubbles. To calculate this force local liquid prop- erties are required, which have to be derived from volume averaged liquid properties.

In addition to the momentum transfer between both phases, the liquid volume fraction $i_{l}$ of a computational cell has to be calculated from the volume occupied by the bubbles present in the cell under consideration and the volume of the computational cell:

$$
i_{i}=1-\frac{\sum_{i} V_{h i}}{V_{\text {cell }}} .
$$

In calculating this liquid volume fraction it is very important to account for bubbles overlapping with more than one computational cell. The volume occupied by these bubbles has to be divided over the respective computational cells.

The momentum transfer from the bubbles to the liquid per unit volume GL dispersion, $\Phi$, is just the opposite of the momentum transfer rate due to drag. lift and virtual mass forces exerted by the liquid on the bubbles present in the computational cell under consideration. Thus, $\Phi$ can be calculated from

$$
\Phi V_{\text {eell }}=\sum_{i}\left(\mathbf{F}_{D i}+\mathbf{F}_{V M i}+\mathbf{F}_{L i}\right)
$$

where the magnitude of the various forces is obtained from the discrete bubble submodel.

In order to calculate the force acting on a bubble from eq. (1) local values of the pressure, of the liquid velocity, of the partial and substantial derivatives of the pressure and of the partial and substantial derivatives of the liquid velocity have to be available at the position of the bubble. However, these Eulerian variables and their derivatives are only known at discrete nodes in the computational domain. Therefore, an area-weighted averaging technique is used to obtain these local values from the values of the Eulerian variables at the four nodes surrounding the bubble. With reference to Fig. 2, the local value of a quantity $f$ can be calculated using

$$
f\left(\mathbf{r}_{i}\right)=\frac{1}{\mathrm{~d} x \mathrm{~d} y_{n}} \sum_{1}^{+} A_{n} t_{n}
$$

with $f_{n}$ being some Eulerian quantity at node $n$, and $A_{n}$ representing an area equal to

$$
\begin{aligned}
& A_{1}=(\mathrm{d} x-\dot{\delta} x)(\mathrm{d} y-\dot{\partial} y) \\
& A_{2}=\delta x(\mathrm{~d} y-\dot{\delta} y) \\
& A_{3}=(\mathrm{d} x-\delta x) \dot{y} y \\
& A_{4}=\delta x \dot{\partial} y
\end{aligned}
$$

\subsection{Boundary conditions}

The boundary conditions required to solve the model equations are incorporated into the model using the flag matrix concept. This concept, also used by Kuipers et al. (1993), allows boundary conditions to be set for each individual computational cell. Using this flag matrix concept a variety of boundary conditions can be set by specifying the value of the cell flag $f l(i, j)$. In Table 1 an overview of the various cell flags 


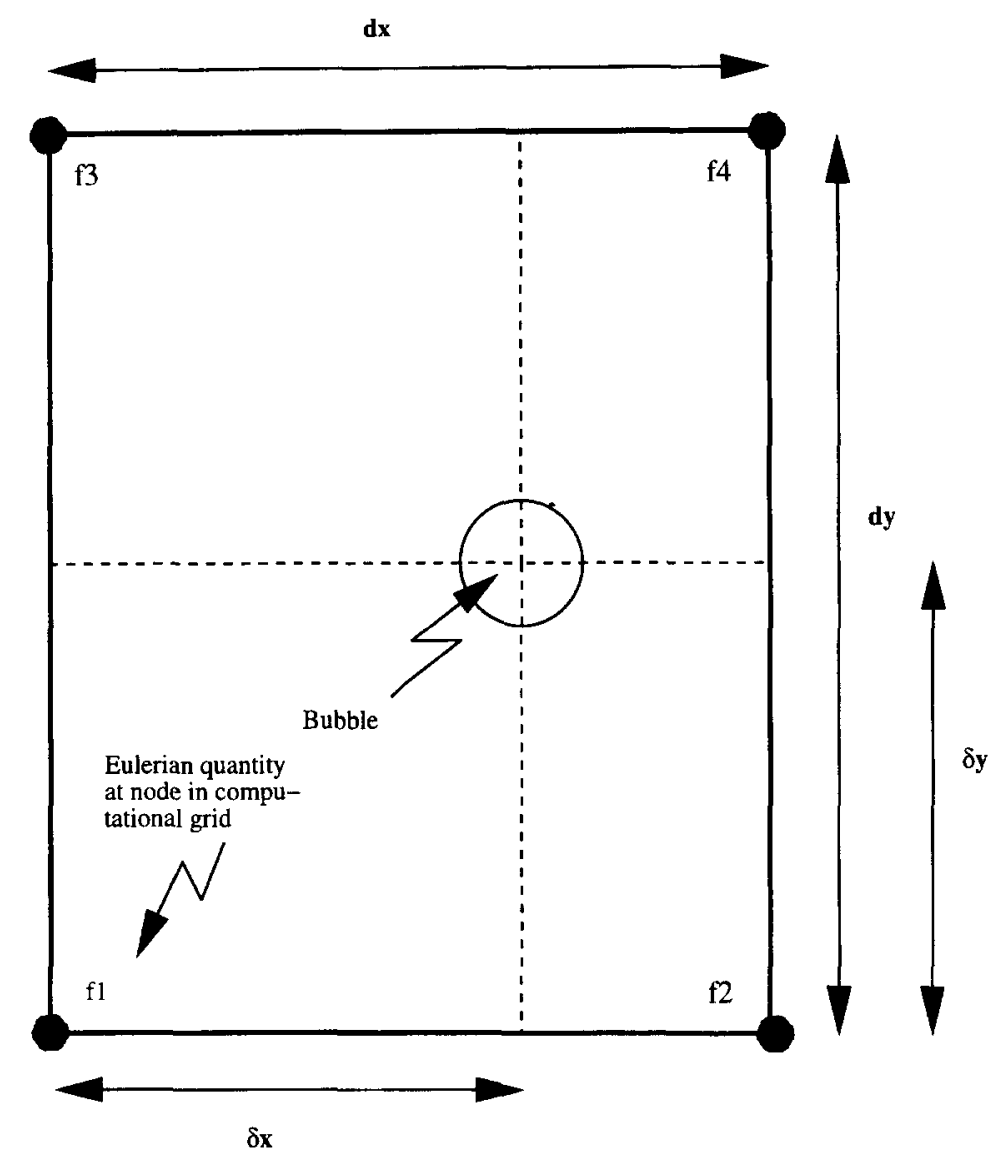

Fig. 2. Area weighting of the four values at the Eulerian nodes surrounding a bubble to obtain the value of the quantity under consideration at the position of the bubble in the computational domain.

Table 1. Cell flags and corresponding cell types used in defining boundary conditions

\begin{tabular}{cl}
$f l(i, j)$ & \multicolumn{1}{c}{ Physical meaning of cell $(i, j)$} \\
\hline 1 & Interior cell, no boundary conditions have to be specified \\
2 & Impermeable wall, free slip boundary \\
3 & Impermeable wall, no slip boundary \\
4 & Fluid-phase influx cell, normal velocity has to be specified \\
5 & Prescribed pressure cell, free slip boundary \\
6 & Continuous outflow cell, free slip boundary \\
7 & Impermeable floor, free slip boundary \\
8 & Impermeable floor, no slip boundary \\
9 & Corner Cell, no boundary conditions have to be specified \\
\hline
\end{tabular}

and the corresponding boundary conditions is given. Figure 3 depicts a typical grid layout used in many of the simulations presented in this paper.

The boundary conditions for the discrete part of the model are essentially provided by the collision routine which accounts for wall-bubble interactions.

\section{NUMERICAL SOLUTION}

In the second and third section of this paper the physics of our model have been described in detail.
This model has been implemented in a computer code, called LeBuc, written in C. A simplified flowsheet of the computer code is presented in Fig. 4. The computer code takes consecutive steps in time, during each of which the code calculates the forces acting on the bubbles present in the system, the new bubble velocities and their new positions, taking into account possible collisions between bubbles. Finally, the code calculates the new liquid velocity field using an algorithm also used by Kuipers et al. (1993). At the end of 


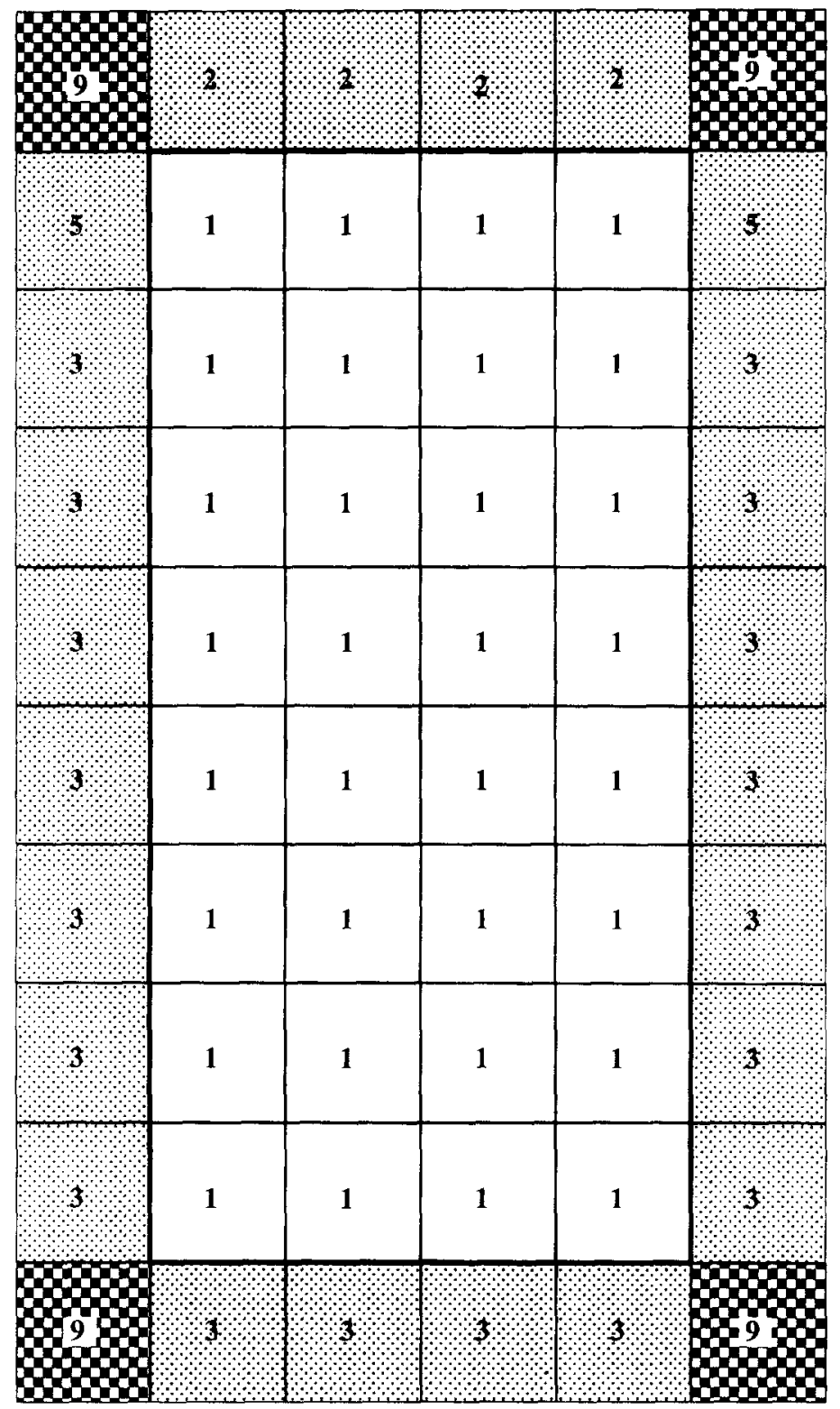

Fig. 3. Typical grid layout for a leBuc simulation. The cell tlags depicted in this figure are explained in Table 1.

each timestep various user specified data in several file formats can be saved.

On average calculating $1 \mathrm{~min}$ of the time-dependent behaviour of a typical bubble column containing several thousand bubbles requires two and a half to $3 \mathrm{~h}$ dedicated CPU time on a Silicon Graphics Indigo $^{2}$ workstation. The CPU time depends on the size of the column and the number of bubbles present in the bubble column. A twofold increase in the number of bubbles present in the bubble column (which can be achieved by doubling the superficial gas velocity) roughly doubles the CPU time required to calculate one minute of real-time operation of the bubble column.

\section{RESULTS AND DISCUSSION}

In the previous sections we have presented a discrete bubble model for a gas-liquid bubble column. The model incorporates both a bubble-bubble interaction model and a fundamental description of the bubble dynamics. In this section the model will be compared to experimental data reported in the literature by various workers. In addition, the model will be used to simulate the time-dependent behaviour of a bubble column. Specifically, both start up behaviour and the effect of column aspect ratio on the two-phase flow will be studied. As the model incorporates a detailed description of the bubble dynamics, the relative 


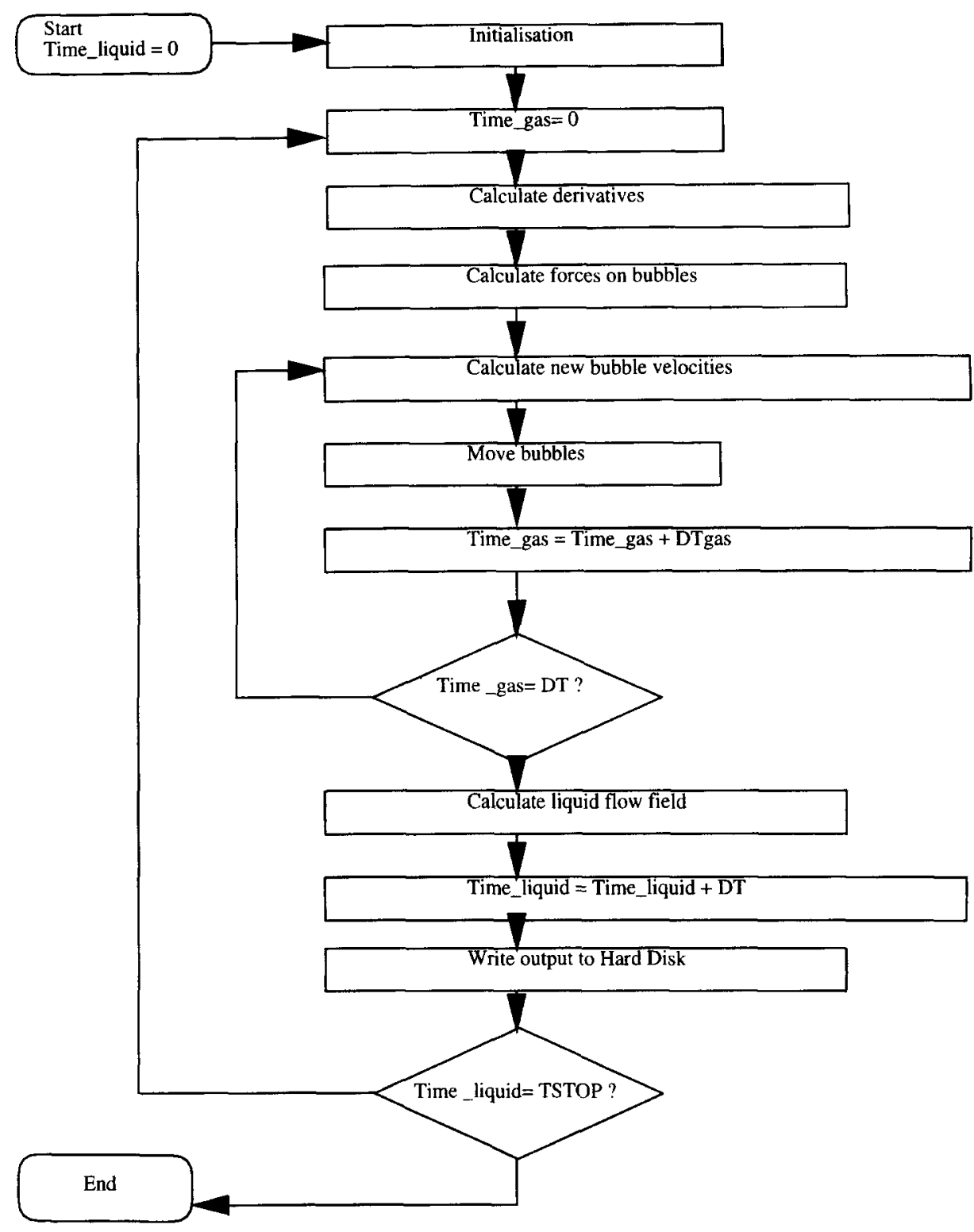

Fig. 4. Flowchart of the leBuc computer code.

importance of the various forces acting on a bubble will also be investigated.

\subsection{Terminal rise velocity of a single bubble}

As a first test case for our model the terminal rise velocity of a single bubble as calculated from a force balance will be compared to the velocity predicted by our model. For a bubble rising with a steady velocity through a quiescent liquid the buoyancy force just equals the sum of gravity force and drag force. From this force balance the bubble's steady rise velocity can be derived:

$$
v_{\text {rise }}=\sqrt{\frac{\frac{8}{3}\left(\rho_{g}-\rho_{l}\right) R_{b} g}{C_{D} \rho_{l}}} .
$$

The drag coefficient $C_{D}$ is calculated using the standard drag curve [eq. (6)]; including this equation into eq. (24) yields an implicit equation in $\mathbf{v}_{\text {rise }}$ which can be solved numerically. This yields a terminal rise velocity of $20.8 \mathrm{~cm} \mathrm{~s}^{-1}$ for a $2 \mathrm{~mm}$ air bubble in water.

Our two-dimensional gas-liquid model was also used to determine the terminal velocity of a single bubble rising in a liquid. We therefore simulated the rise of a single $2 \mathrm{~mm}$ air bubble in a bubble column of $1.5 \mathrm{~m}$ in height and $0.50 \mathrm{~m}$ in width (computational conditions are listed in Table 2). At a height of $1 \mathrm{~m}$ above the gas distributor the bubble appeared to have a steady rise velocity of $20.9 \mathrm{~cm} \mathrm{~s}^{-1}$, which agrees well with the theoretically calculated bubble rise velocity.

\subsection{Comparison with experimental data reported by} Becker et al. (1995)

Becker et al. conducted experiments in a pseudotwo-dimensional bubble column; the results of two of 
Table 2. Parameters used to calculate terminal rise velocity of a single air bubble in water using the Eulerian/Lagrangian model

\begin{tabular}{cccc}
\hline Bubbles and liquid & & \multicolumn{3}{c}{ Column } \\
\hline Bubble diameter & $2(\mathrm{~mm})$ & Height & $1.50(\mathrm{~m})$ \\
Gas flow rate & Width & $0.50(\mathrm{~m})$ \\
Density gas & $1.2\left(\mathrm{~kg} \mathrm{~m}^{-3}\right)$ & Number $x$-cells & 50 \\
Density liquid & $1000\left(\mathrm{~kg} \mathrm{~m}^{-3}\right)$ & Number $y$-cells & 100 \\
Liquid shear viscosity & $1 \times 10^{-3}(\mathrm{Pas})$ & DT & $5.0 \times 10^{-3}(\mathrm{~s})$ \\
Gas & Air & & \\
Liquid & Tap water & & \\
\hline
\end{tabular}

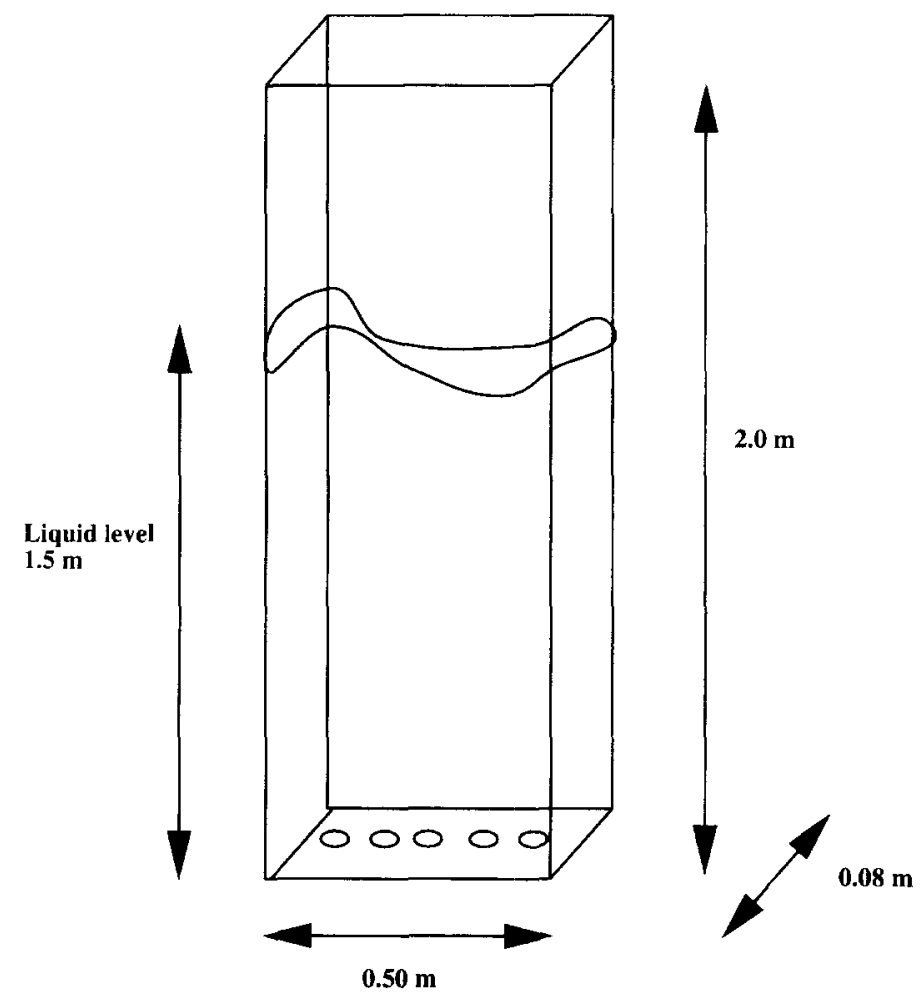

Fig. 5. Experimental set up used by Becker $t$ t al. (1995). Liquid level is $1.5 \mathrm{~m}$.

their experiments, i.e. with a small and a large superficial gas velocity, will be compared with computational results obtained from our model. The experimental setup used by Becker et al. is depicted in Fig. 5; consists of a two-dimensional bubble column (width: $50 \mathrm{~cm}$, height: $2 \mathrm{~m}$ and depth: $8 \mathrm{~cm}$ ). The column is equipped with a gas distributor consisting of five, individually controllable porous plates.

Becker et al. fed gas (air) to the column (containing water) through only one of the five porous plates during their experiments. This plate is located $15 \mathrm{~cm}$ from the left wall of the column and consists of a $40 \mathrm{~mm}$ plastic disc with an active pore size of $15 \mu \mathrm{m}$. Becker et al. reported data on the liquid velocity field obtained for two different superficial gas velocities. Liquid velocities were measured using Laser Doppler Anemometry and microturbine anemometry techniques.
Our model was used to simulate a two-dimensional cross section of the bubble column under consideration. The gas bubbles, with a diameter of $2 \mathrm{~mm}$, were generated in a regular pattern at the gas distributor where the rate of bubble generation was calculated from the specified gas flow rate. Experimental conditions and numerical parameters are listed in Table 3.

5.2.1. Comparison between model and experiment for large superficial gas velocity. Becker et al. describe in detail the flow structure which they have observed in their bubble column. They reported the existence of a gross circulation flow over the whole height of the column which firmly pushes the bubble swarm against the left wall of the column. As the bubble swarm reaches the free surface, it is influenced by a secondary vortex which develops at the upper left corner. This causes part of the swarm to flow downwards near the 
Table 3. Parameters used in model simulation of experiments reported by Becker et al.

\begin{tabular}{cccc}
\hline Bubbles and liquid & \multicolumn{3}{c}{ Column } \\
\hline Bubble diameter & $2\left(\mathrm{~mm}^{2}\right.$ & Height & $1.50(\mathrm{~m})$ \\
Gas flow rate (high) & $8.0\left(\mathrm{~min}^{-1}\right)$ & Width & $0.50(\mathrm{~m})$ \\
Gas flow rate (low) & $1.6\left(\mathrm{~min}^{-1}\right)$ & Number $x$-cells & 50 \\
Density gas & $1.2\left(\mathrm{~kg} \mathrm{~m}^{-3}\right)$ & Number $y$-cells & 100 \\
Density liquid & $1000\left(\mathrm{~kg} \mathrm{~m}^{-3}\right)$ & Orifice width & $4.0 \times 10^{-2}(\mathrm{~m})$ \\
Liquid shear viscosity & $1 \times 10^{-3}(\mathrm{Pas})$ & DT & $5.0 \times 10^{-3}(\mathrm{~s})$ \\
Gas & Air & & \\
Liquid & Water & & \\
\hline
\end{tabular}

right wall of the column. The liquid is observed to flow upwards in the vicinity of the bubble swarm and downwards along the right wall of the column.

A number of snapshots visualising the developing flow as calculated by our model are shown in Fig. 6.* It can be seen from these figures that the calculated flow structure is similar to that reported by Becker $e t$ al. Our model predicts the development of a powerful liquid circulation which pushes the bubble swarm firmly towards the left wall of the column. This strong liquid circulation is induced by the large number of bubbles which rise through the column. At the upper part of the left wall a secondary vortex develops which changes the path of the bubble swarm and causes a part of the bubbles to flow downwards near the right side of the column, as reported by Becker et al. These bubbles are more or less trapped in the downward liquid flow and form a rotating bubble-cloud which does not change in size considerably.

Becker $e t$ al. reported an essentially steady-state flow structure. In this respect the results obtained with our model differ from those obtained by Becker $e t$ al. The calculated flow structure appears to be more oscillatory due to the interaction of the bubble plume with vortices that develop near the upper left corner. These vortices move up and down along the upper part of the column's left wall. The aforementioned difference between model and experiment is most likely due to the two-dimensional nature of our model.

The computed time-averaged liquid velocity field is depicted in Fig. 7, it clearly indicates the existence of a gross liquid circulation with liquid upflow near the left wall of the column and downflow near the opposite wall of the column. The velocity field depicted in Fig. 7 was averaged over the first $300 \mathrm{~s}$ of simulation time.

5.2.2. Comparison between model and experiment for small superficial gas velocity. At lower superficial

\footnotetext{
*The bubbles indicated in the figures presented in this paper have been exaggerated in size in order to make them visible to the reader.
}

gas velocities Becker et al. observed a remarkably different flow pattern in their bubble column. The bubble swarm now moves upwards in a meandering manner. Several liquid circulation cells were reported which change their location and size continuously. The flow was observed to be highly dynamic. Longtime measurements of the vertical liquid velocity at a point $900 \mathrm{~mm}$ above the distributor and $35 \mathrm{~mm}$ from the left wall of the column, revealed a period of oscillation of the vertical velocity of approximately $41 \mathrm{~s}$.

Figure 8 shows snapshots of the behaviour of the bubble plume at these lower gas velocities as calculated from our model. The same meandering behaviour as was reported by Becker et al. is clearly reproduced by our model. From video representation of the computational results it can clearly be seen that the meandering behaviour of the plume is caused by a vortex that develops at the upper left corner of the column. Subsequently, this vortex moves downwards along the left wall of the column and disappears at the bottom of the column. This process continues with the development of a new vortex at the upper left corner of the column. This continuous vortex development and disappearance together with the weak circulating liquid flow allows for the bubble plume to meander.

Figure $8(f)$ depicts the vertical liquid velocity as a function of time at the point chosen by Becker et al. for their measurements of the vertical liquid velocity. The mean vertical liquid velocity predicted by the model is $-0.077 \mathrm{~m} \mathrm{~s}^{-1}$ which differs from the result obtained by Becker $e t$ al., who measured a mean vertical liquid velocity of $-0.038 \mathrm{~m} \mathrm{~s}^{-1}$. The period of oscillation of the vertical liquid velocity calculated by the model is approximately $30 \mathrm{~s}$. The smaller period of oscillation found in our computation and the difference between the calculated and the measured mean vertical liquid velocity is most likely due to the two-dimensional nature of our model which does not account for the front and back wall of the 'real-life' pseudotwo-dimensional bed used by Becker et al. To study this effect the extension of the present two-dimensional model to a full three-dimensional model is 

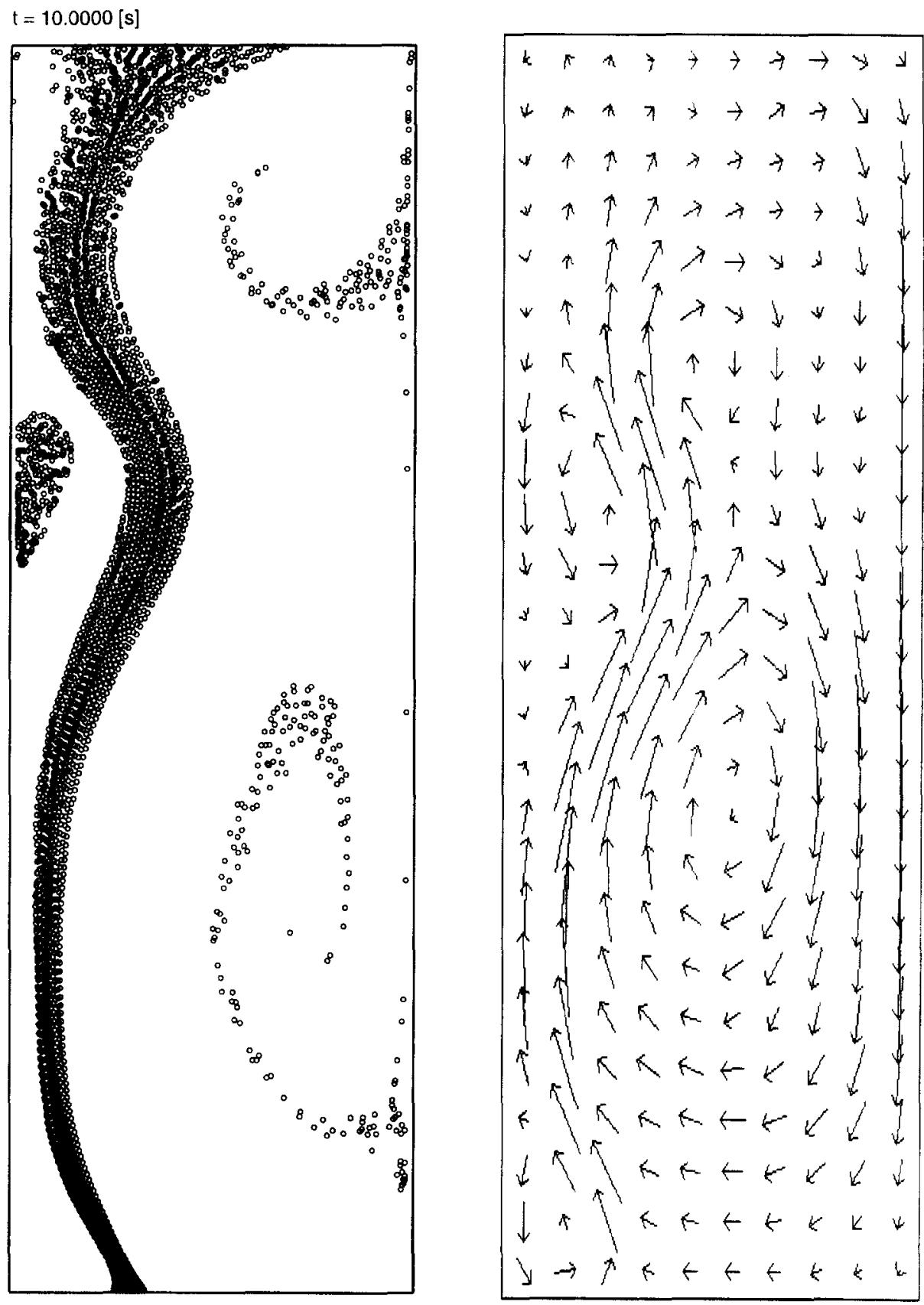

(a)

Fig. 6. Bubble positions and instantaneous liquid velocities at (a) $10 \mathrm{~s}$; (b) $30 \mathrm{~s}$; and (c) $60 \mathrm{~s}$ after start of gas feed. Simulation of experiment by Becker et al. (1995) for high superficial gas velocity. Gas flow rate $=8.01 \mathrm{~min}^{-1}$. Additional conditions are listed in Table 3 . 

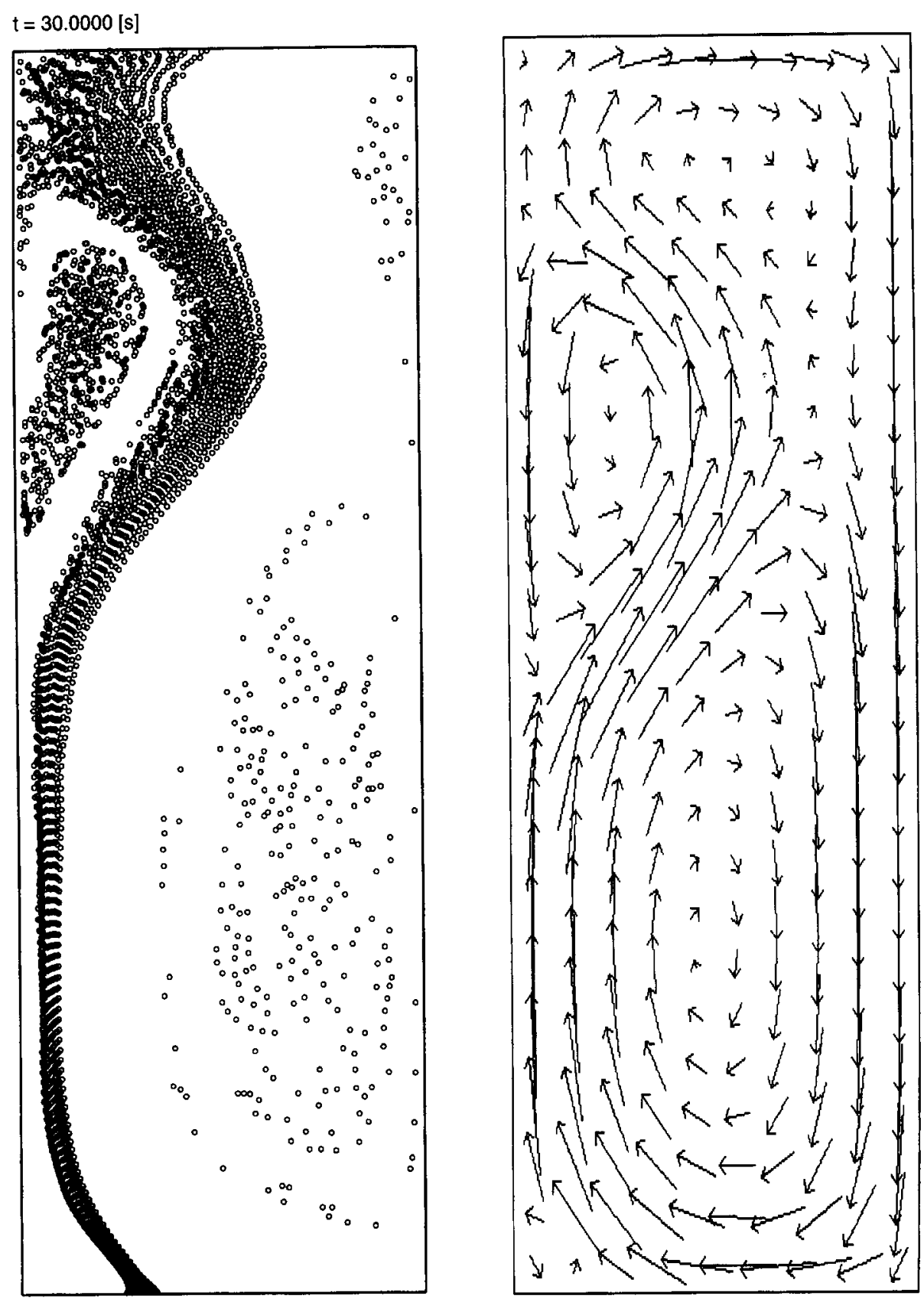

(b)

Fig. 6(b). 

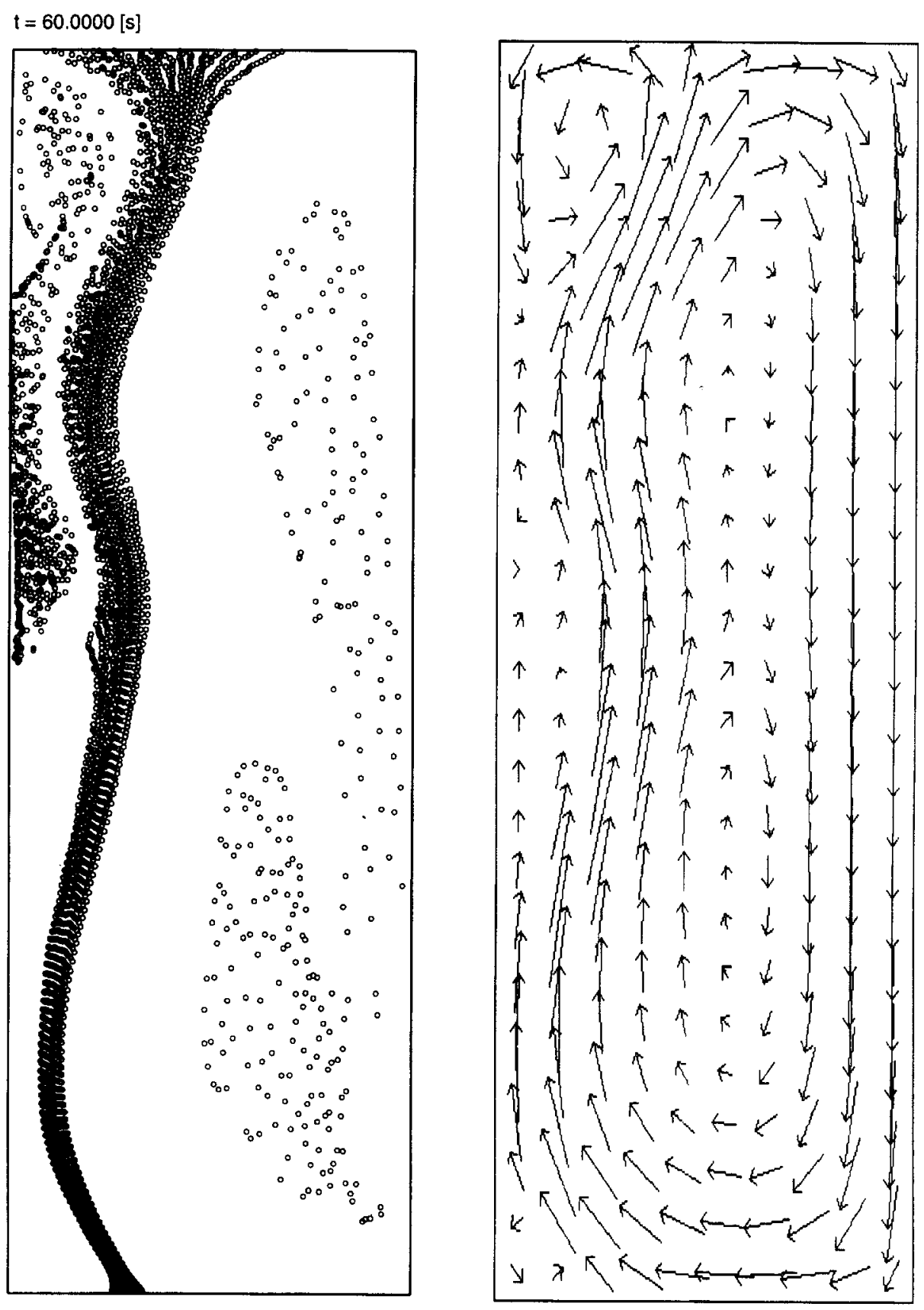

(c)

Fig. 6(c). 


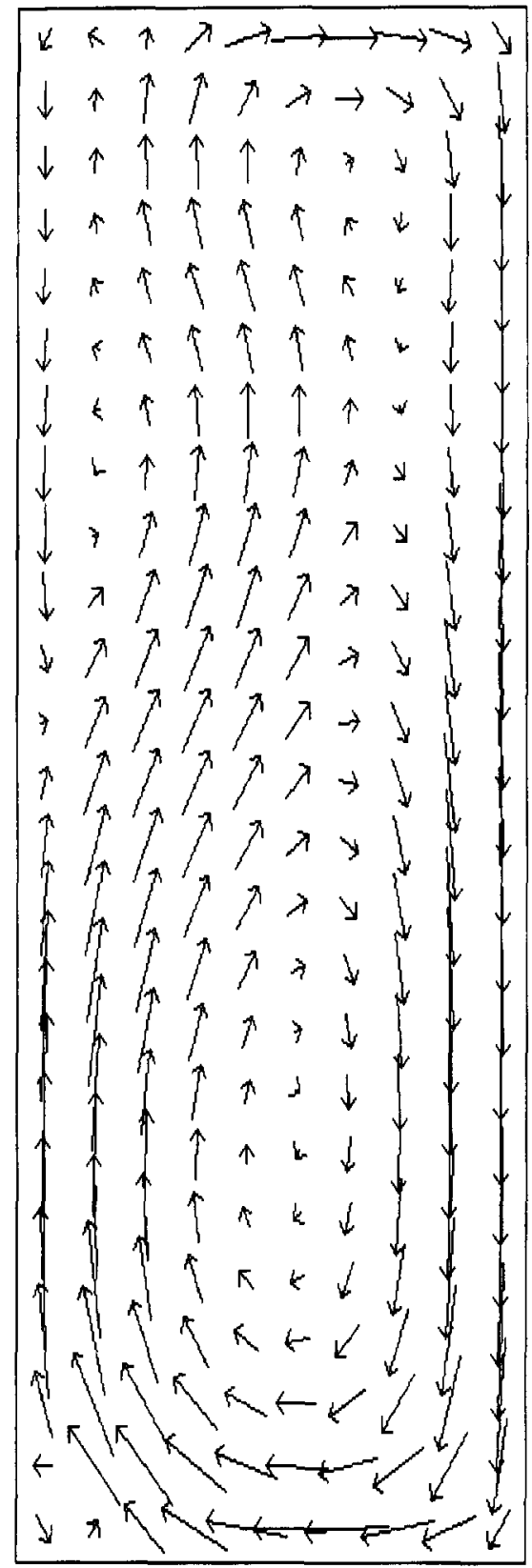

Fig. 7. Time-averaged liquid velocity field $(0-300 \mathrm{~s})$. Simulation of experiment by Becker et al. (1995) for high superficial gas velocity. Additional conditions are listed in Table 3.

required. This will be an important aspect of our future work.

5.2.3. Comparison with experiments at small superficial gas velocity using a simplified model version. In recent years a number of publications have appeared in the literature reporting Eulerian/Lagrangian models for gas-liquid bubble columns. Some of these models neglect the momentum transfer between the gas bubbles and the liquid phase and evaluate the bubble velocity as a simple sum of the local liquid velocity and an empirically determined slip velocity. The coupling between the phases is usually achieved through the effective density of the mixture.

In order to asses the validity of these simplifications we have used a simplified version of our model to simulate the behaviour of the bubble plume at the small superficial gas velocity. The model incorporated a very simple description of the bubble dynamics, i.e. a bubble velocity which is the sum of the local liquid velocity and a constant slip velocity. Momentum transfer between the phases was neglected. Figure 9 depicts the behaviour of the bubble plume at the low gas velocity. From these snapshots, it is clear that a simplified description of the bubble dynamics and a neglect of momentum transfer between the phases cannot account for the highly time-dependent meandering behaviour of the bubble plume as observed by Becker et $a l$. and as calculated from our model incorporating detailed bubble dynamics and momentum transfer between the phases. These detailed bubble dynamics and the momentum coupling between the phases are, in our opinion, essential for an accurate description of the time-dependent behaviour of a gas-liquid bubble column.

\subsection{Effect of aspect ratio of a bubble column on its hydrodynamic hehaviour}

There is an extensive literature on fluid circulation inside bubble columns and its relation to the aspect ratio of the bubble column. Well-known models are the Gulfstream model introduced by Freedman and Davidson (1969) and the multiple circulation cell model due to Joshi and Sharma (1979). The effect of the aspect ratio of the bubble column on the liquid circulation was studied experimentally by Chen $e t a l$. (1989). They studied liquid circulation in bubble columns with varying liquid depth by streak photography. Chen et al. conducted experiments in two different pseudo-two-dimensional beds at constant superficial gas velocity.

Chen et al. observed the Gulfstream mode of circulation in bubble columns with an aspect ratio smaller than or equal to unity. This Gulfstream model consists of two vortex cells opposite to each other, with liquid upflow in the middle of the column and liquid downflow near both walls. If the aspect ratio exceeds unity, the flow pattern observed is that of multiple, staggered circulation cells. The circulation pattern proposed by Joshi and Sharma (1979) was not observed.

Our model was used to study the effect of the aspect ratio of the bubble column on the liquid circulation pattern. Bubble columns with two different aspect ratios have been studied at a constant gas flow rate of $1.5 \mathrm{ml} \mathrm{s}^{-1}$. Figures 10 and 11 show the calculated instantaneous liquid velocity profiles and bubble positions at different time levels in bubble columns with aspect ratio's of 2 and 4, respectively. It can be seen that the flow structure clearly changes with the aspect ratio of the column. For an aspect ratio equal to 2 the Gulfstream type of flow pattern is observed. It 

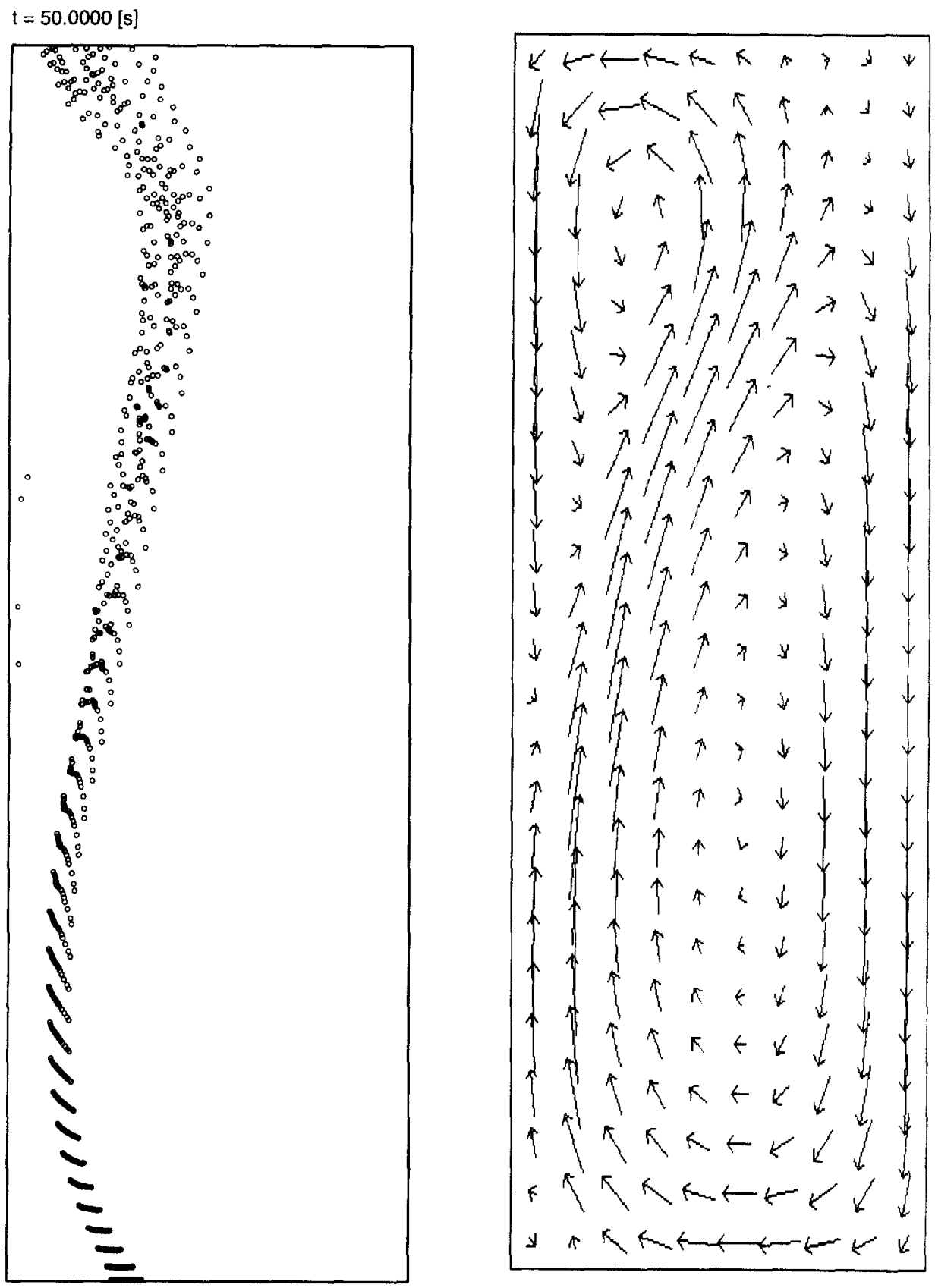

(a)

Fig. 8. Bubble positions and instantaneous liquid velocities at (a) $50 \mathrm{~s}$; (b) $60 \mathrm{~s}$; (c) $70 \mathrm{~s}$; (d) $80 \mathrm{~s}$; (e) $90 \mathrm{~s}$ after start of gas supply. (f) Vertical liquid velocity at $900 \mathrm{~mm}$ above gas distributor and $35 \mathrm{~mm}$ from left wall ats function of time $(0-300$ s). Simulation of experiment by Becker et al. (1995) for low superficial gas velocity. Gas flow rate $=1.6 \mathrm{l} / \mathrm{min}$. Additional conditions are listed in Table 3 . 

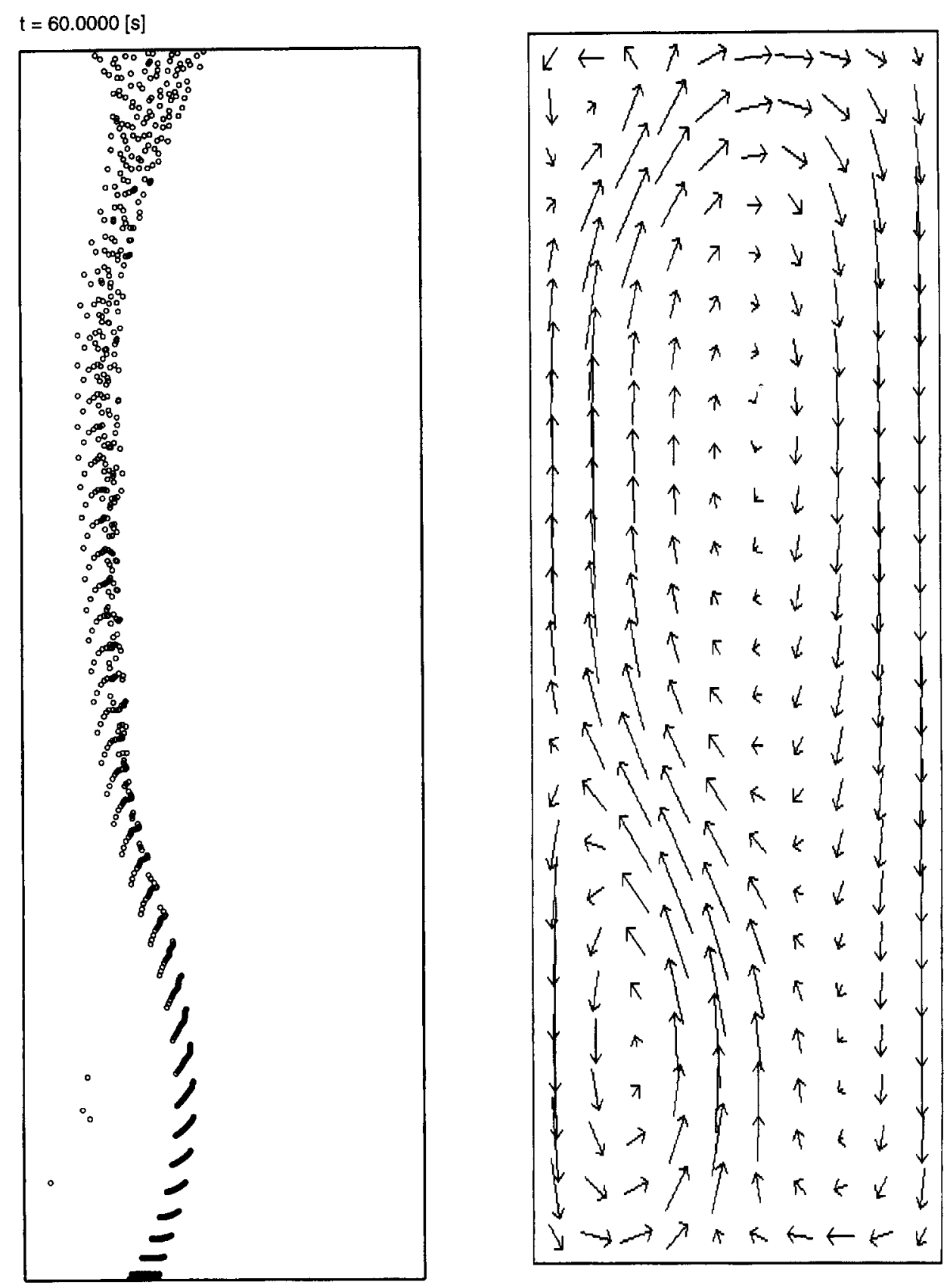

(b)

Fig. 8(b). 

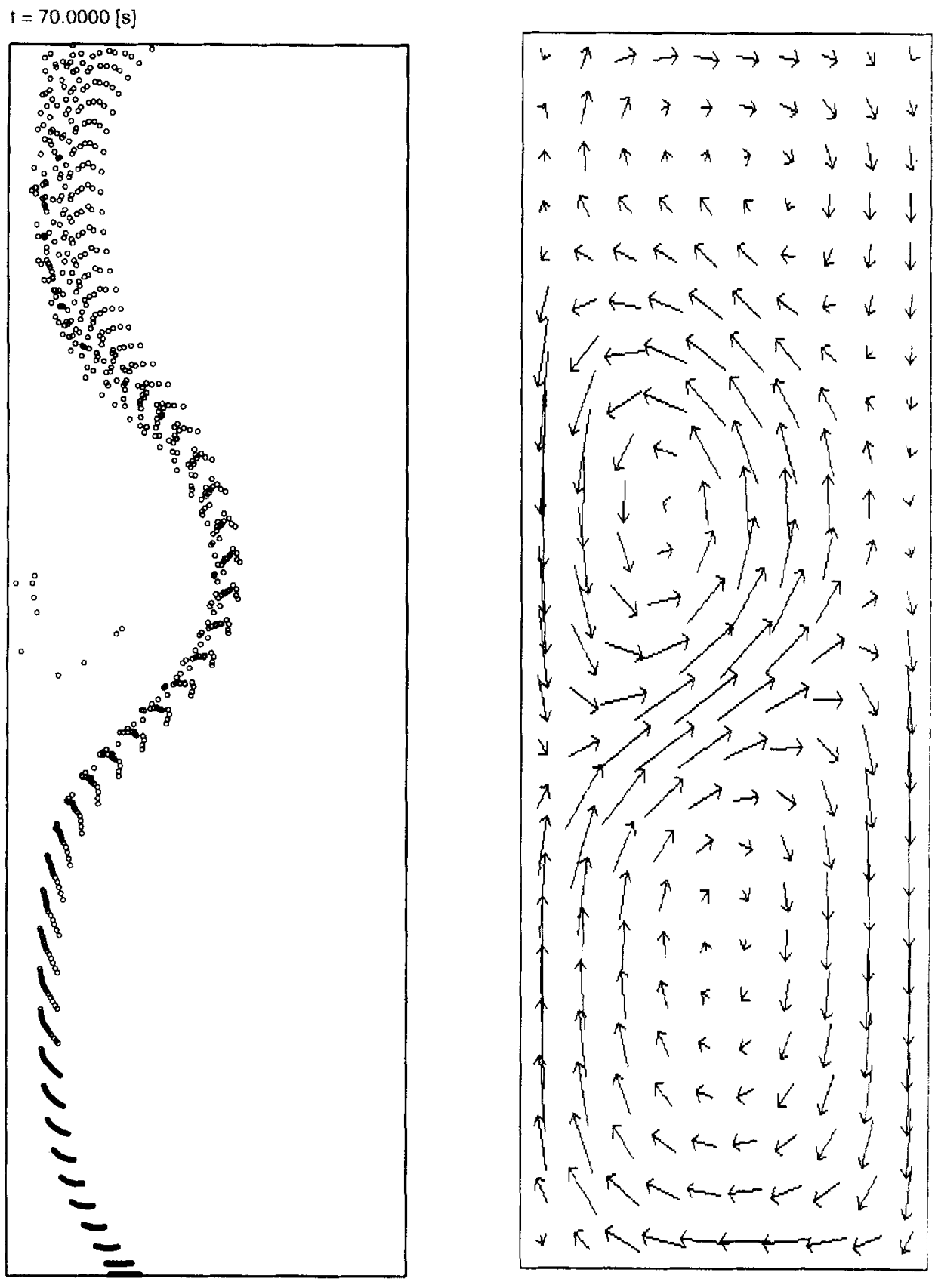

(c)

Fig. $8(\mathrm{c})$ 

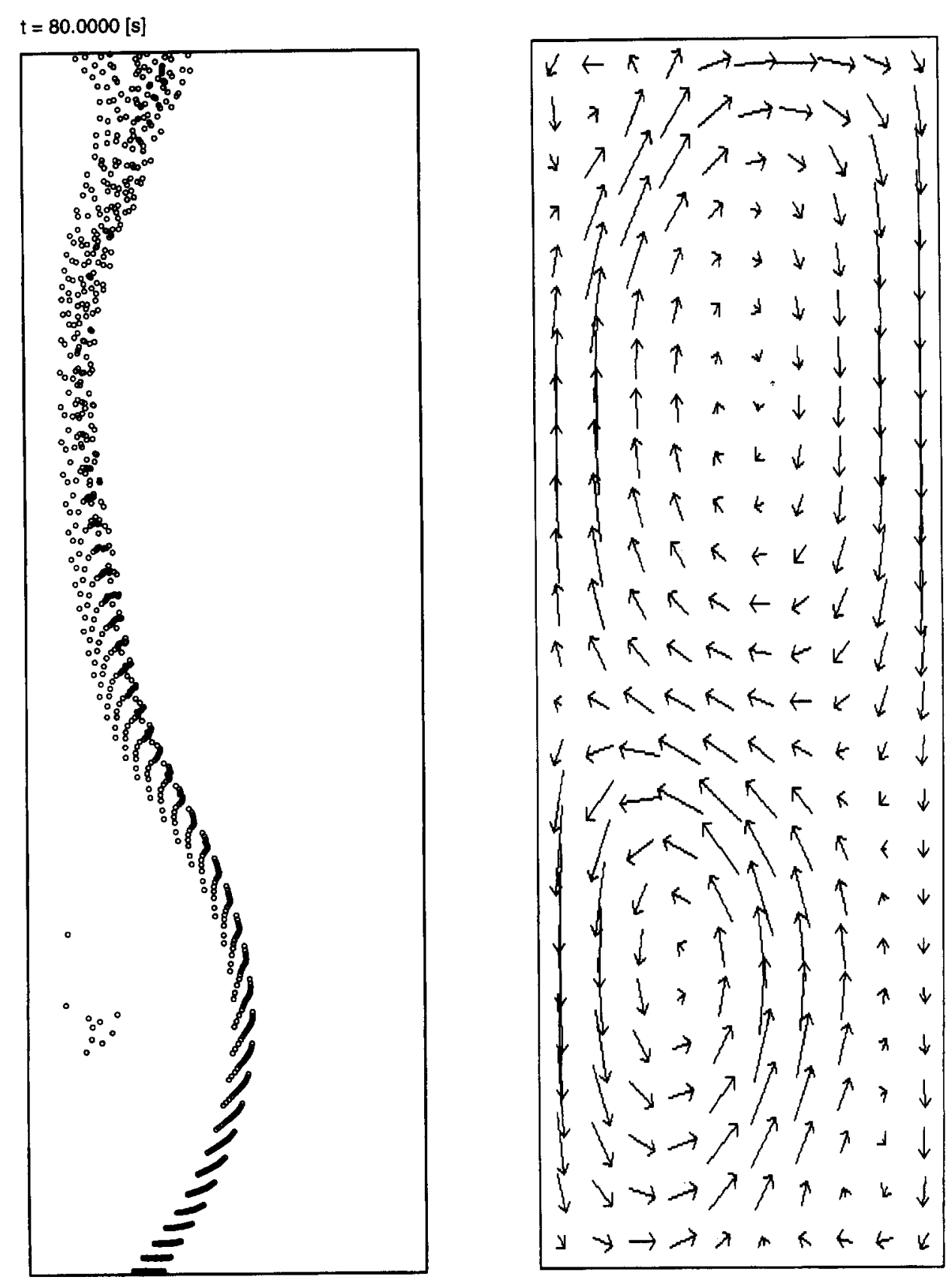

(d)

Fig. 8(d). 

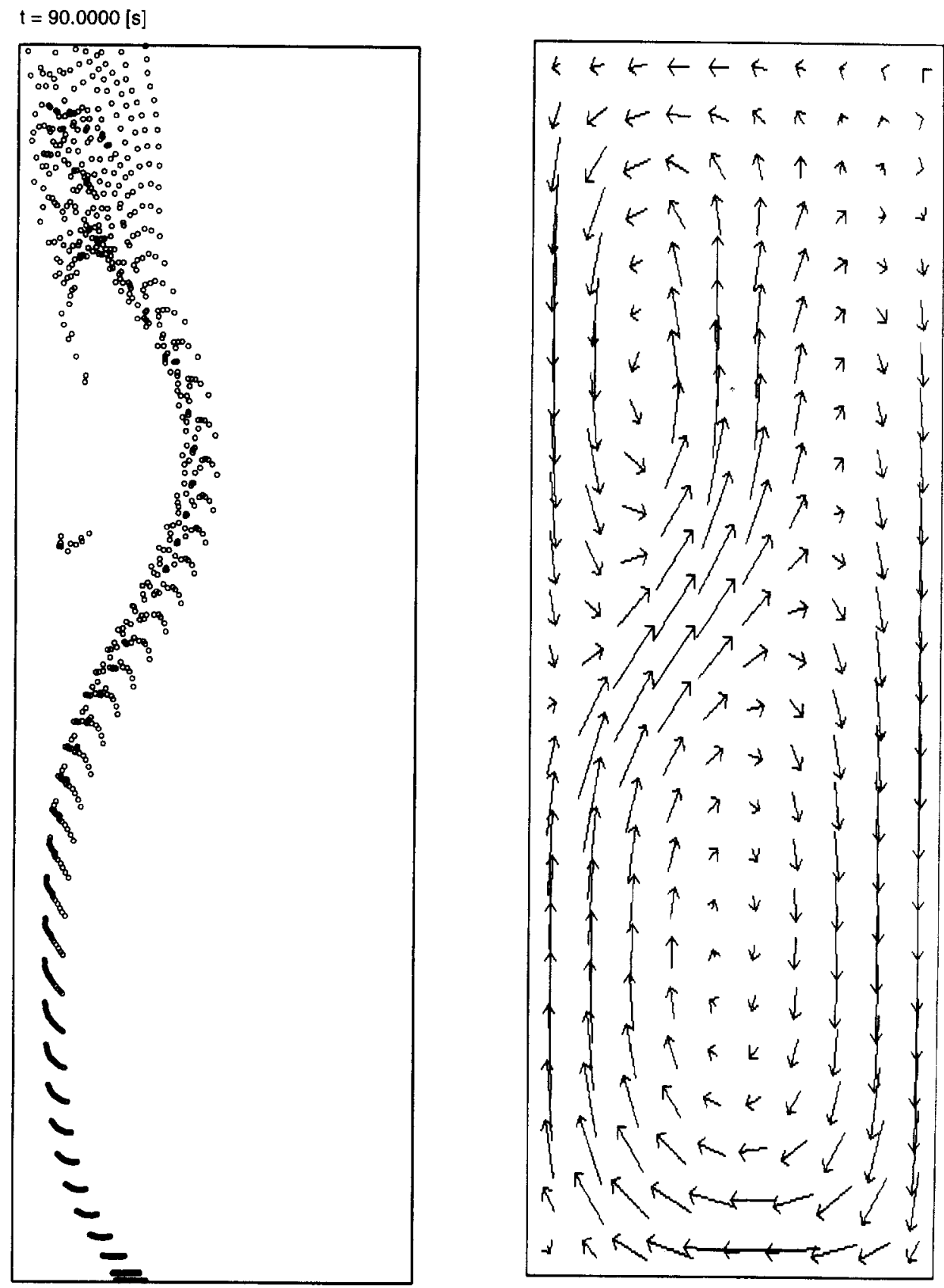

(e) 


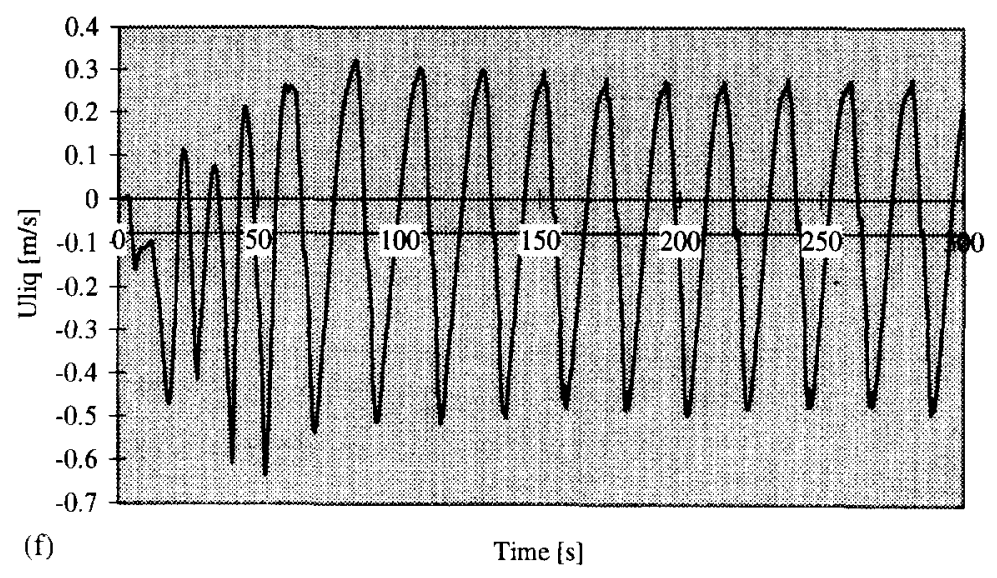

Fig. $8(\mathrm{f})$

can be seen that both initially and after several minutes of simulation time the bubble plume rises in between two circulation cells. For an aspect ratio of 4 , the behaviour of the bubble column is quite different. The bubble plume rises through the liquid in a meandering manner. The plume is influenced by multiple vortices that develop at the free surface; these vortices possess a staggered orientation with respect to each other. In this respect our model compares well with Chen's experimental observations, although Chen et al. predict a transition from the Gulfstream flow pattern to the meandering time-dependent flow pattern at an aspect ratio of one. Our results also indicate that the structure of the two-phase gas-liquid flow depends on the aspect ratio of the column.

Figure 12 shows the time-averaged liquid velocity profile in the bubble column with an aspect ratio of four. The velocities were averaged over the time interval from 0 to $60 \mathrm{~s}$. Clearly, the well-known liquid velocity profile with upflow in the column centre and downflow near the column walls is obtained in this case. The velocity pattern is observed to be highly symmetric. From Figs 11 and 12 it is clear that the time averaged liquid velocity profile does not capture the time-dependent behaviour of the flow in a bubble column with an aspect ratio equal to four.

\subsection{The importance of the added mass force and the lift force}

One of the key features of the model presented in this paper is the detail with which the bubble dynamics is described. In Section 2 it has been assumed that the force acting on a spherical bubble rising in a liquid is composed of separate and uncoupled contributions from pressure gradient, gravity, drag force, added mass force and the lift force. These last two forces are of particular importance despite the fact that they are not usually incorporated in fluid dynamic descriptions of gas-liquid two-phase flow.

The added mass force appears to be very important in the vicinity of the gas distributor. In the model presented in this paper, bubbles are assumed to grow at the distributor until a specified radius has been attained; at that point in time the bubbles detach from the distributor. Near the distributor the bubbles accelerate under the influence of buoyancy. The only force restricting this acceleration to realistic values is the added mass force; drag forces are not yet important because after detachment the bubble velocity is still very small. Without added mass forces included in the model, the bubble acceleration reaches an unrealistic value because of the relatively strong buoyant forces and the small mass of the bubbles. The omission of the added mass force causes the model to become unstable. From our model simulations and careful analysis of the computed data, we conclude that added mass forces are essential for an accurate description of the behaviour of small, spherical gas bubbles in the vicinity of the gas distributor region of the bubble column.

The importance of the lift force acting on the bubbles has also been investigated. The time-dependent behaviour of the bubble column with a length-todiameter ratio of 4 as studied in Section 5.3 has also been calculated without lift forces acting on the bubbles. Figure 13 depicts the instantaneous bubble positions. From this figure and from Fig. 11(a) it can clearly be seen that lift forces cause the bubble plume to expand, as the shear-induced lift force acting on the bubbles in the bubble plume is directed towards the walls of the column. The bubble plume seen in Fig. 13 remains narrow, the bubbles do not spread out over the cross section of the column. The behaviour seen in Fig. 13 is not realistic; lift forces acting on bubbles are therefore important, and must be included in an Eulerian/Lagrangian model of a bubble column in the homogeneous regime. However, the exact value of the lift coefficient is still to be investigated.

\subsection{Start up of a bubble column}

A problem of interest for practical application of bubble columns is concerned with their start-up 
$t=10.0000[s]$

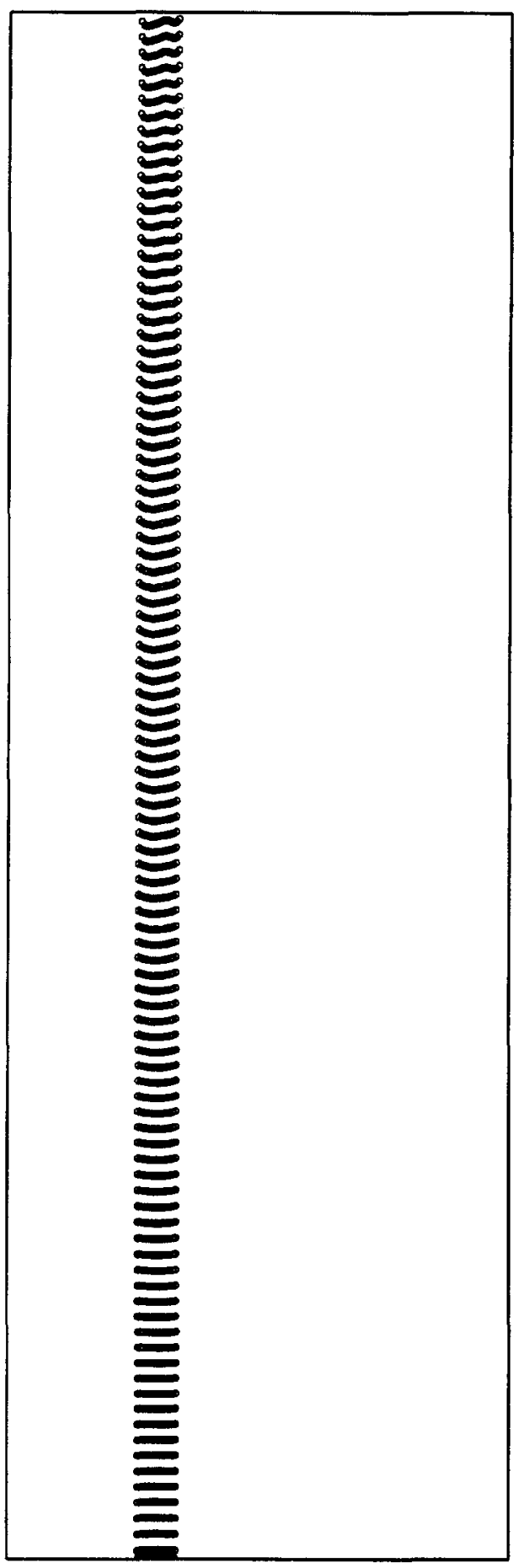

$t=60.0000[s]$

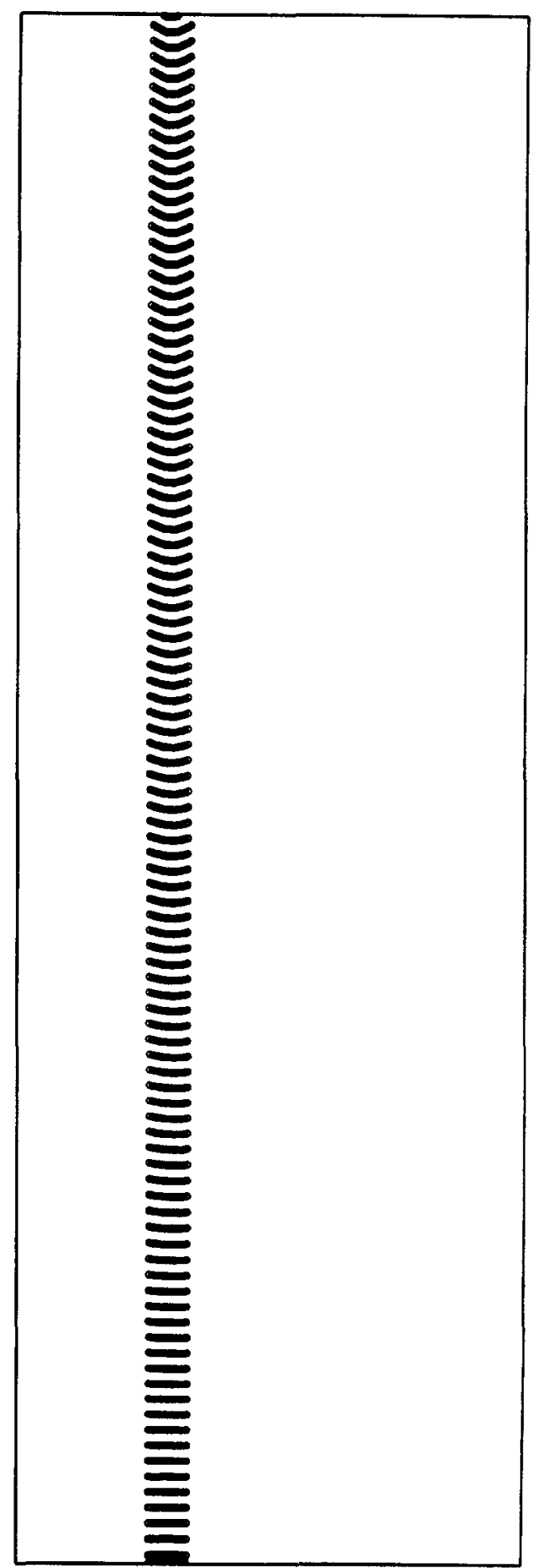

Fig. 9. Instantaneous bubble positions at two different time levels. Simulation performed with a simplified version of our model which does not incorporate a detailed description of the bubble dynamics and does not account for momentum exchange between both phases. Conditions used in the simulation are derived from Becker et al.'s experiment for low superficial gas velocity (Table 3). 

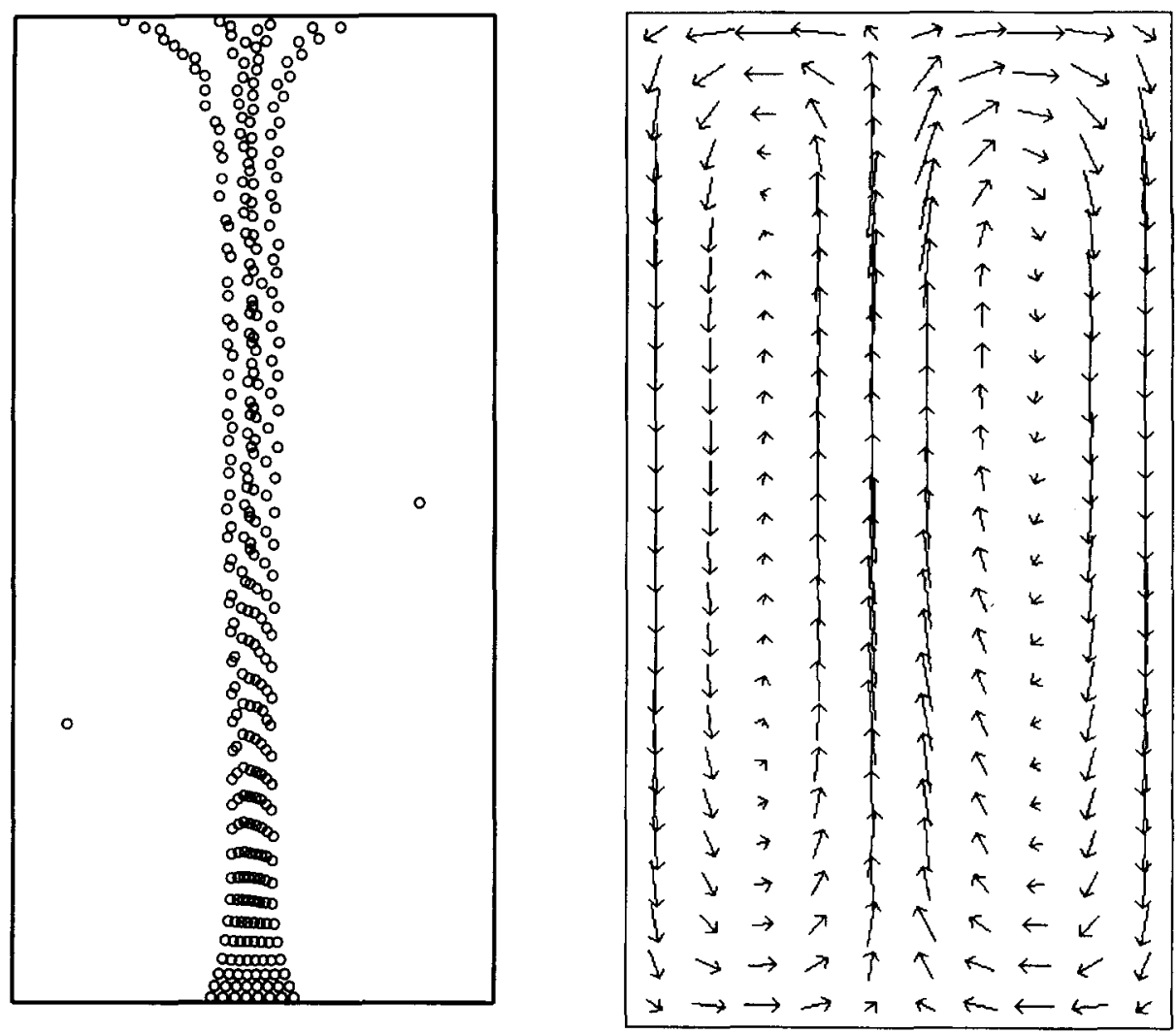

$\mathrm{t}=30.0000[\mathrm{~s}]$
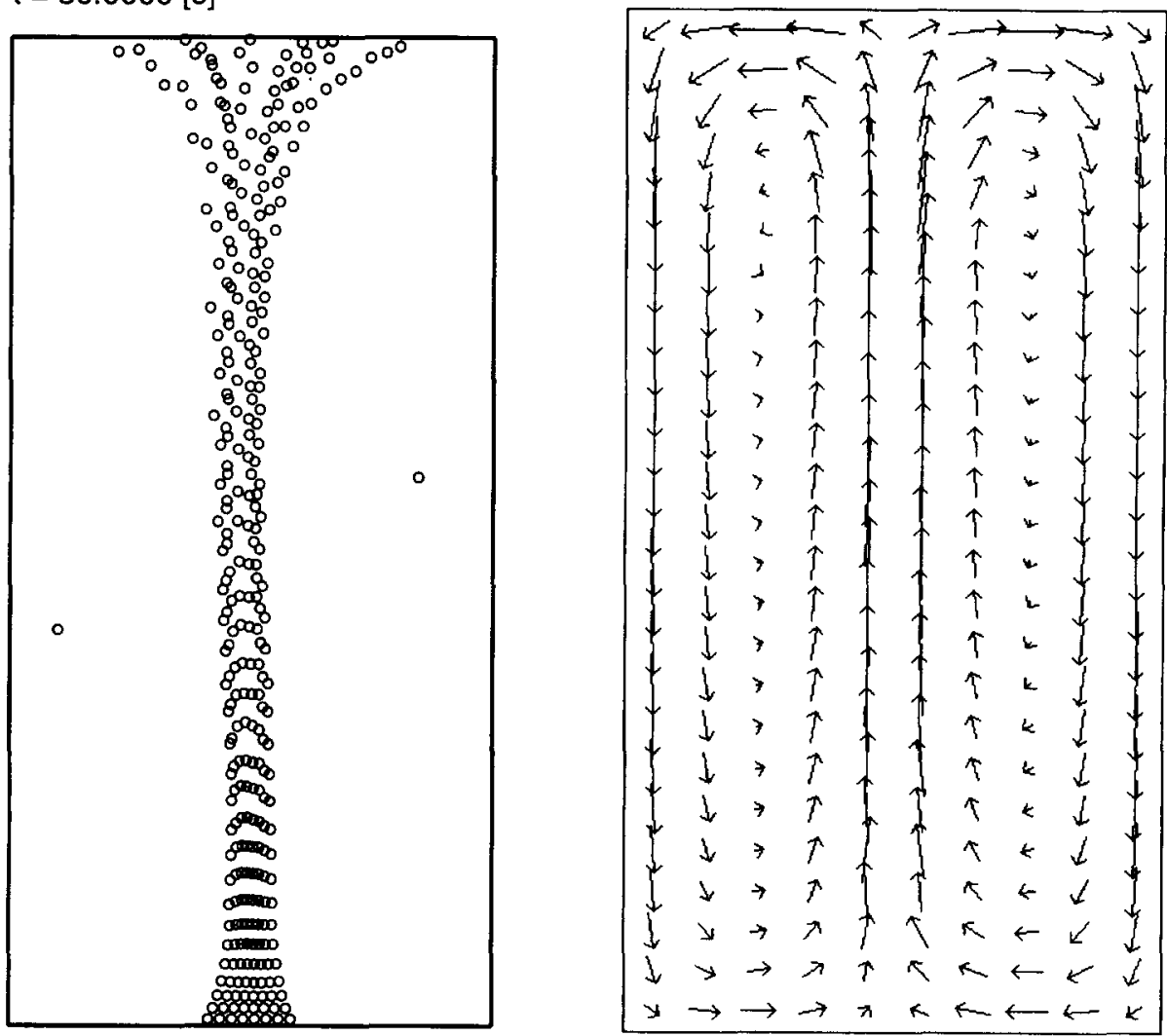

(a)

Fig. 10 (a) Bubble positions and instantaneous liquid velocity field in a bubble column with an aspect ratio of 2 at two different time levels. Air-water system. Gas flow rate $=6.0 \mathrm{ml} \mathrm{s}^{-1}$. Grid: $20 \times 50$ computational cells. (b) Bubble positions in a bubble column with an aspect ratio of 2 at two different time levels. Air-water system. Gas flow rate $=6.0 \mathrm{ml} \mathrm{s}^{-1}$. Grid: $20 \times 50$ computational cells. 
$t=300.0000[s]$

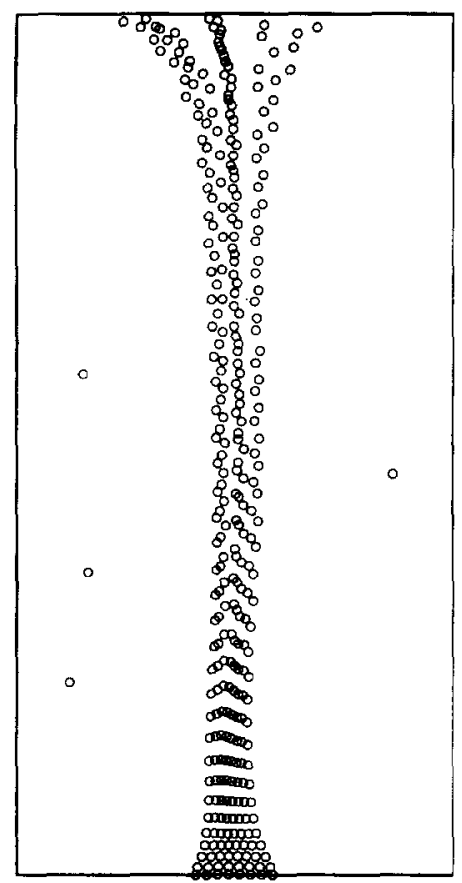

(b)

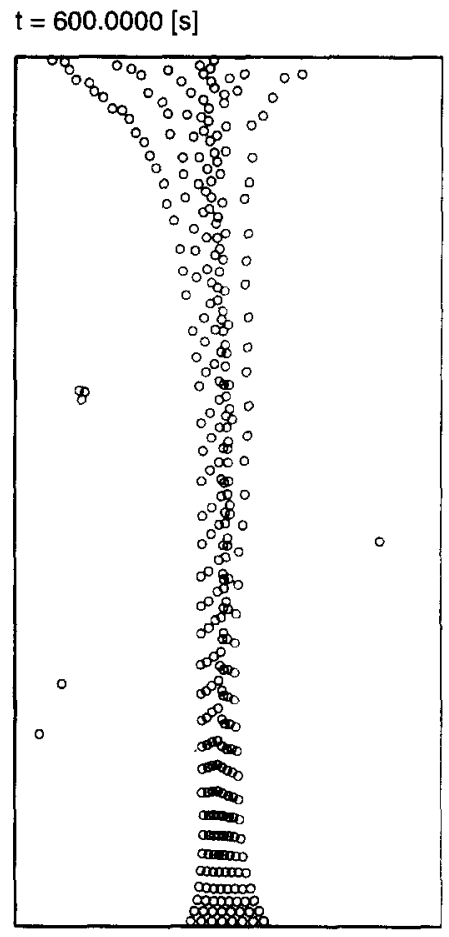

Fig. 10(b).

behaviour. This problem has, to our kinowledge, not been investigated theoretically yet. Our model has therefore been used to simulate the hydrodynamics during start up of a bubble column. As a test problem the behaviour of a bubble column aerated through two gas distributors was simulated; the total gas flow rate used equalled $4.0 \mathrm{ml} \mathrm{s}^{-1}$. Two cases were considered; first gas was supplied to a gas distributor at the right-hand side of the column centre and after $1 \mathrm{~s}$ the gas supply to a distributor at the left-hand side was started; this is termed the 'right-first' scenario. The second start up procedure (i.e. the 'left-first' scenario) is just the mirror image of the first procedure; the left-hand-side gas distributor was started $1 \mathrm{~s}$ before the right-hand-side gas distributor was started.

Figure 14 compares the bubble positions for both cases $30 \mathrm{~s}$ after start up. Video representation of the computational results indicated only minor changes in the depicted gas-liquid flow pattern after these initial $30 \mathrm{~s}$. This can also be seen from Fig. 15 where the flow pattern after 10 min of simulation time has been shown for the 'left-first' scenario.

It is therefore concluded that Fig. 14 depicts the flow structure ultimately obtained in the bubble column. It can clearly be seen that there is a difference in the flow structure obtained in either scenario. The outcome of the 'right-first' simulation indicates that both bubble plumes rise along the right-side wall of the bubble column. A single circulation cell develops near the left wall of the column. The flow pattern for the 'left-first' simulation is just the mirror image of the 'right-first' result, with both bubble plumes rising along the left wall of the column. It can therefore be concluded that the flow pattern obtained in a bubble column depends on the start up procedure of that bubble column; the history of the flow appears to have a profound effect on the flow pattern which is ultimately established.

Of course, the two scenarios considered here correspond to a rather extreme situation but these results indicate some of the complex features which can be encountered in practice.

\section{CONCLUSIONS}

An Eulerian/Lagrangian model for a gas-liquid bubble column has been developed which resolves the two-dimensional, time-dependent motion of small spherical gas bubbles in a liquid. The model incorporates all relevant forces acting on a bubble in a liquid. such as contributions from the pressure gradient in the liquid, drag, added or virtual mass, vorticity in the liquid phase and gravity. A direct bubble-bubble interaction model resembling Hoomans' collision model for gas-fluidized beds has been incorporated in our model. The liquid-phase hydrodynamics are described using the volume-averaged Navier-Stokes equations.

Our model has been validated using experimental data from the literature obtained by various workers. The model was shown to compare well to the experiments conducted by Becker $e t$ al. The behaviour of the 

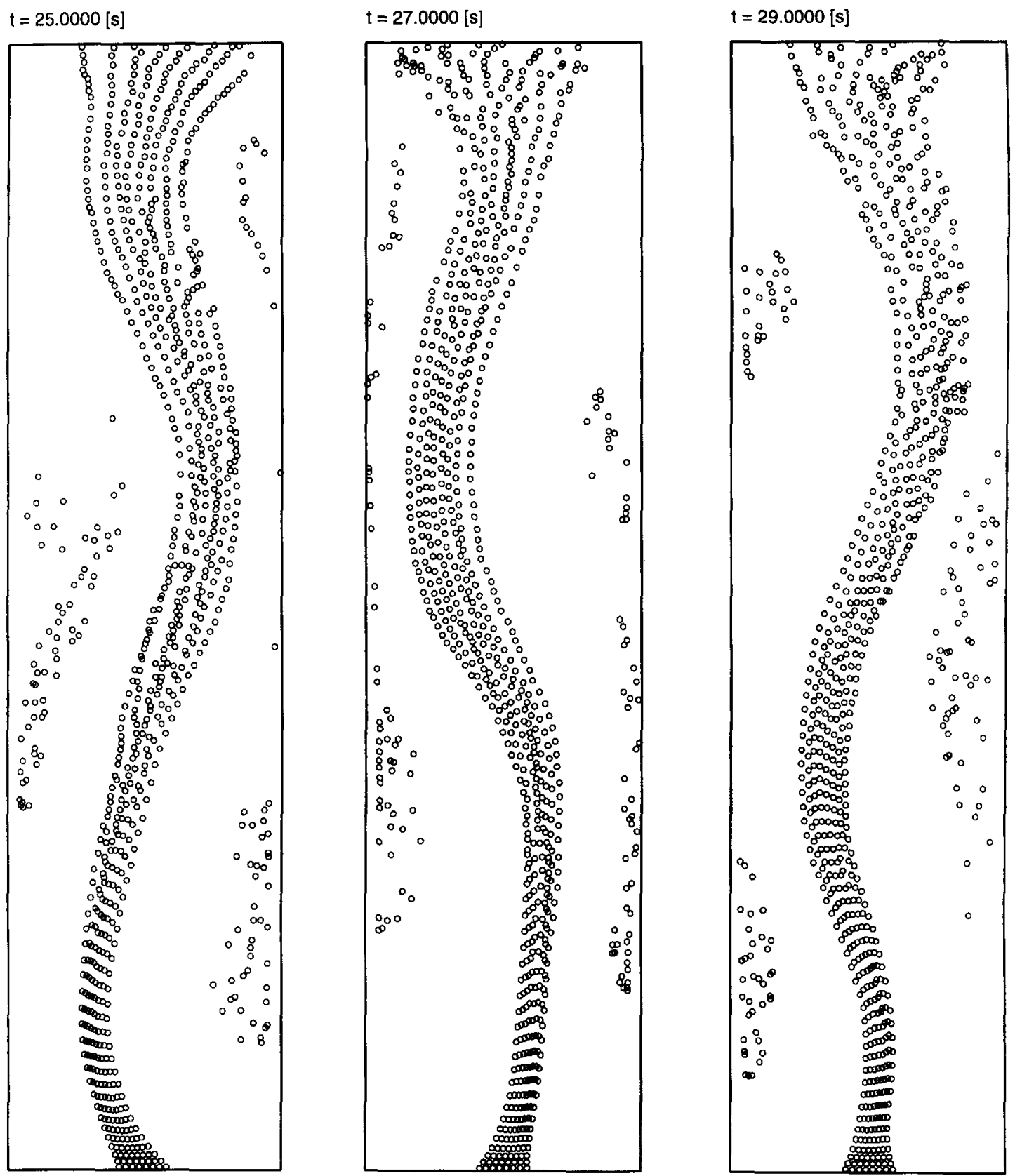

(a)

Fig. 11 (a) Instantaneous bubble positions in a bubble column with an aspect ratio of 4 at three different time levels. Bubbles are depicted somewhat larger as compared to the scale of the column. Air-water system. Gas flow rate $=6.0 \mathrm{ml} \mathrm{s}^{-1}$. Grid: $20 \times 100$ computational cells. (b) Corresponding instantaneous liquid velocities in a bubble column with an aspect ratio of 4 at three different time levels. 


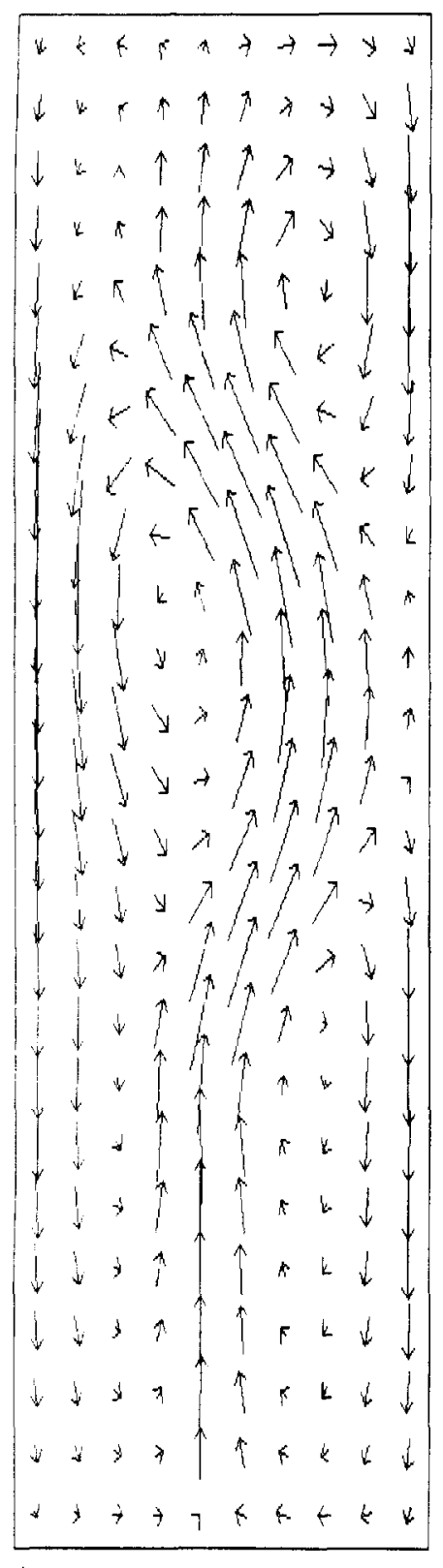

(b)
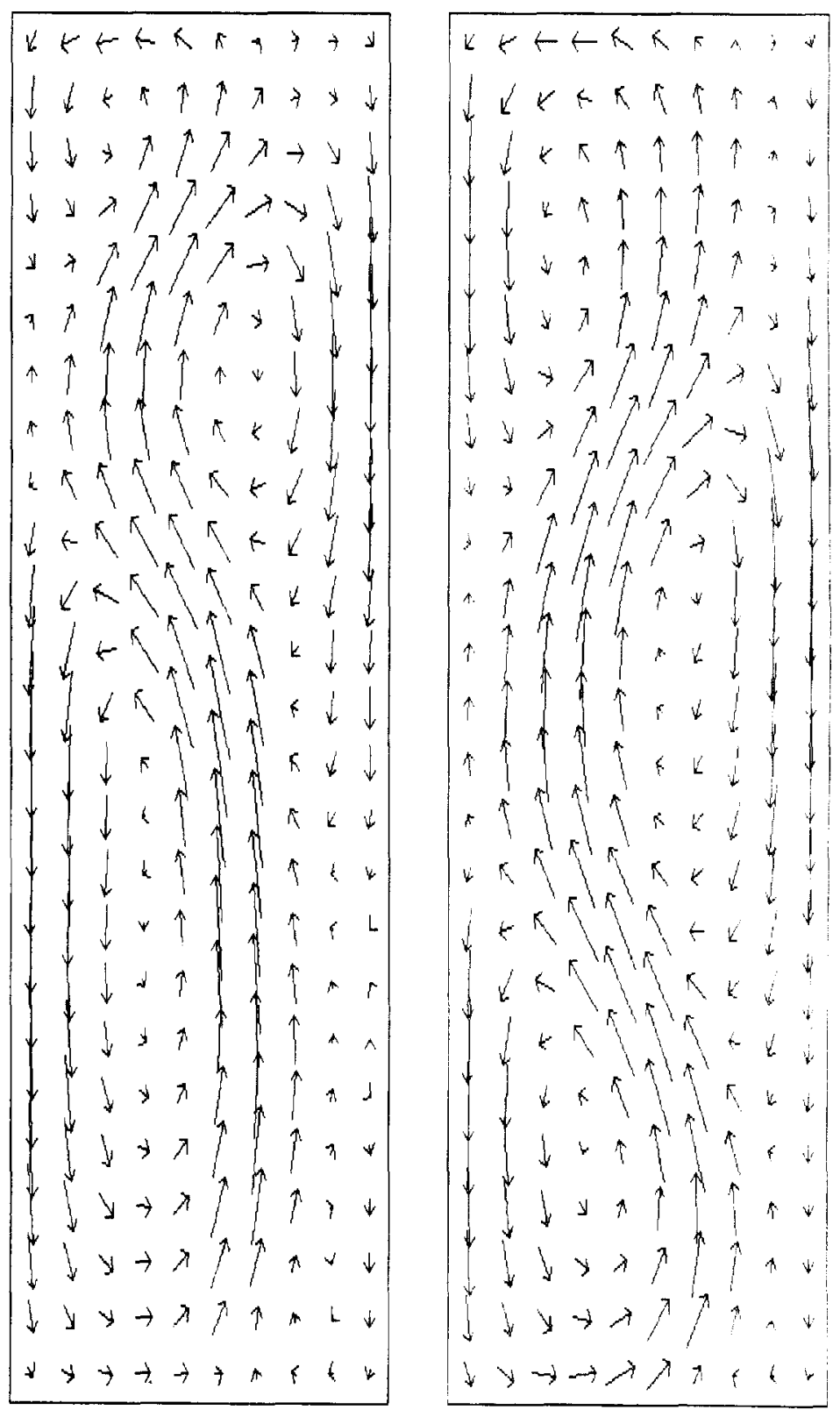

Fig. 1l(b). 


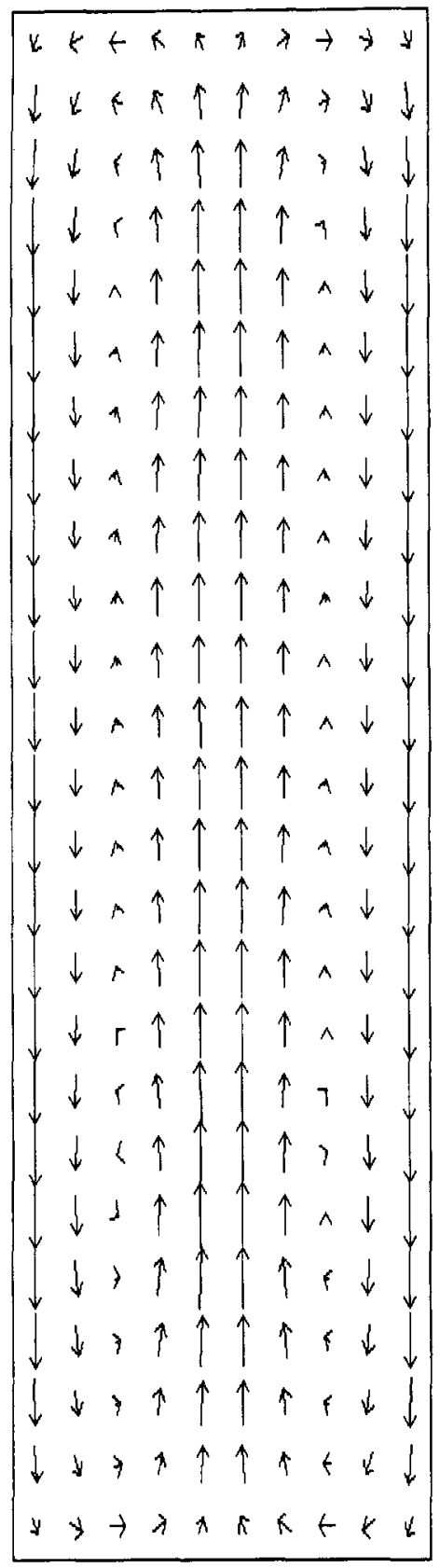

Fig. 12. Time-averaged liquid velocity field in the bubble column with an aspect ratio of 4 . Conditions as listed with Fig. 11. Velocities averaged from 0 to $60 \mathrm{~s}$ after start up of the bubble column.

bubble plume in Becker's two-dimensional bubble column was predicted accurately for both the high superficial gas velocity and the low superficial gas velocity case. The experimentally observed meandering behaviour of the bubble plume at low superficial gas velocities was calculated where the period of oscillation of the bubble plume predicted by the model was found to be of the same order of magnitude as the one observed by Becker et al.

The importance of incorporating a detailed description of bubble dynamics and of incorporating momentum transfer between the gas and the liquid phase into the model was also shown. For the geometry studied by Becker $e t$ al. it was shown that a simplified version of our model, without detailed bubble dynamics and momentum exchange, was not able to predict the experimentally observed, time-dependent gasliquid flow pattern.

The model was also used to investigate the effect of the aspect ratio of the bubble column on the overall liquid circulation pattern. This effect has been studied experimentally by Chen et al. Our computational results indicate a transition in the gas-liquid flow pattern as the aspect ratio of the column changes from two to four. For an aspect ratio equal to two the Gulfstream type of liquid circulation was observed. At an aspect ratio of four however, a highly dynamic liquid flow pattern with multiple vortices was observed. These vortices were observed to be generated at the free surface and found to be positioned staggered with respect to each other. In part, these results support Chen's findings. Chen et al. also observed a transition in the gas-liquid flow pattern from the Gulfstream mode to the highly dynamic multiple vortex mode. However, Chen et al. found this transition to occur at an aspect ratio of one.

One of the key features of the model presented in this paper is the detailed bubble dynamics. The importance of the added mass force and the lift force acting on the bubbles was investigated theoretically. It was found that the added mass force is of particular importance near the gas distributor. Neglecting the added mass force will (initially) cause the bubbles to accelerate at an unrealistic high rate. The lift force acting on the bubbles in the bubble plume is directed towards the walls of the column, and causes the bubble plume to diverge. Without lift forces, the bubbles do not spread out over the cross section of the column, resulting in an unrealistic, narrow bubble plume. It is therefore concluded that lift forces acting on the bubbles must be included in any Eulerian/Lagrangian model of a gas-liquid bubble column operating in the homogeneous regime.

Finally, our model was used to study effects encountered in start up of bubble columns. A model system was chosen to investigate the effect of start up procedure on the steady-state hydrodynamics. In the case one start up procedure the bubble column was initially only aerated via a gas distributor at the right side of the column. After $1 \mathrm{~s}$ gas was also fed to a gas distributor at the left side of the column. The case two start-up procedure was just the mirror image of this first start-up procedure. It was shown that the instantaneous liquid velocity fields ultimately obtained in these two different situations are mirror images of one another. It was also found that the history of the flow had a marked influence on the prevailing flow pattern under pseudo-steady-state conditions. 

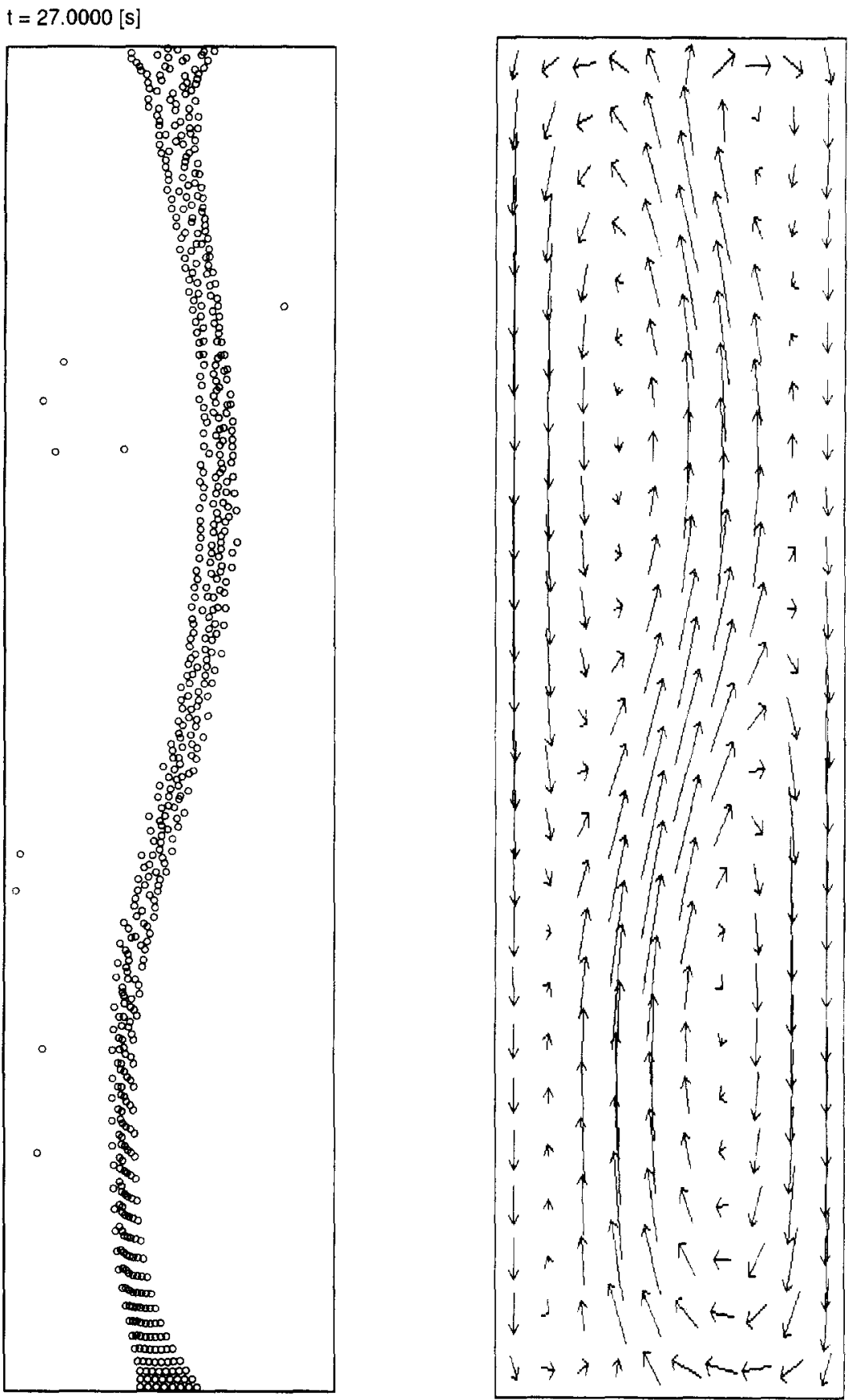

Fig. 13. Instantaneous bubble positions and liquid velocity field at $27 \mathrm{~s}$ after start up in at bubble column with an aspect ratio of 4 . Bubbles are depicted somewhat larger as compared to the scale of the bubble column. Air-water system. Gas flow rate $=6.0 \mathrm{ml} \mathrm{s}^{-1}$. Grid: $20 \times 100$ computational cells. Lift forces are not included in the model. 

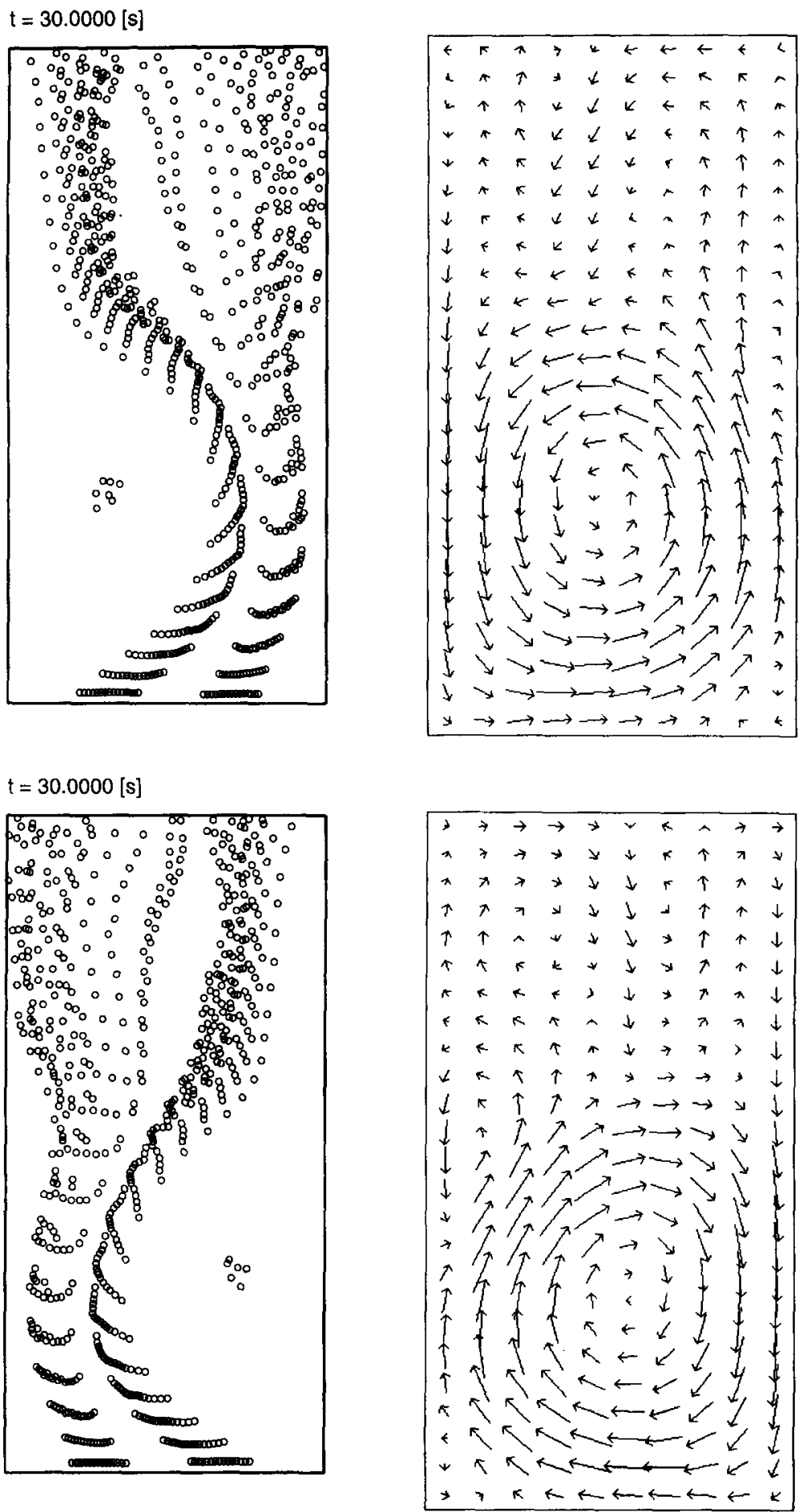

Fig. 14. Bubble positions and liquid velocities at $30 \mathrm{~s}$ after start up for both start up procedures. Top: 'right-first' senario. Bottom: 'left-first' senario. Gas flow rate $=4.0 \mathrm{ml} \mathrm{s}^{-1}$. Grid: $20 \times 50$ computational cells. 

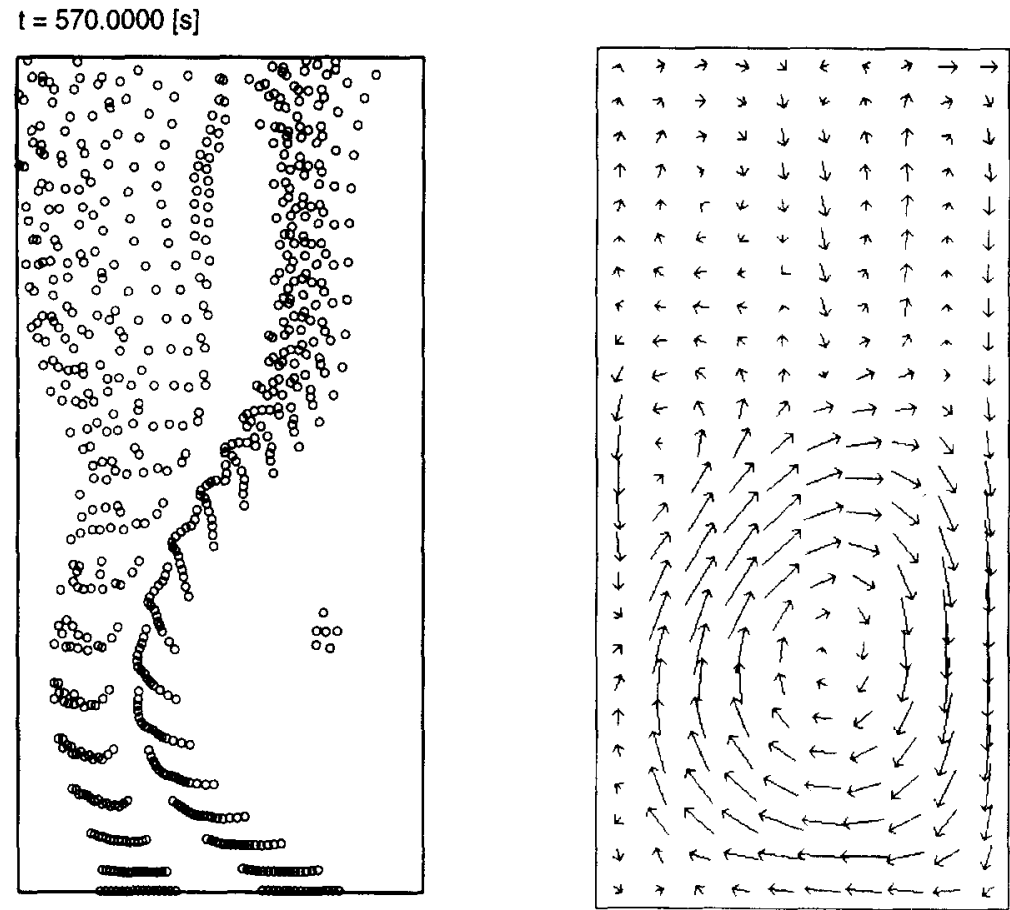

Fig. 15. Bubble positions and liquid velocities at $570 \mathrm{~s}$ after start up for 'left-first' scenario. Gas flow rate $=4.0 \mathrm{ml} \mathrm{s}^{-1}$. Grid: $20 \times 50$ computational cells.

\section{NOTATION}

A area, $\mathrm{m}^{2}$

$C_{D} \quad$ drag coefficient, dimensionless

$C_{L} \quad$ lift force coefficient, dimensionless

$C_{V M} \quad$ virtual mass coefficient, dimensionless

DT time step solver liquid flow field, s

E unit tensor, dimensionless

$\mathbf{F}_{n} \quad$ drag force on a bubble, $\mathrm{N}$

$\mathbf{F}_{G} \quad$ force on bubble due to gravity, $\mathrm{N}$

$\mathbf{F}_{l} \quad$ lift force on bubble, $\mathrm{N}$

$F_{P}$ force on bubble due to pressure gradient, $\mathrm{N}$

$F_{\text {total }}$ total force on a bubble, $\mathrm{N}$

$\mathrm{F}_{V_{M}}$ virtual mass force on bubble, $\mathrm{N}$

g acceleration due to gravity, $\mathrm{m} \mathrm{s}^{-2}$

I kelvin impulse deformable body, Ns

$m_{h} \quad$ mass of a bubble, $\mathrm{kg}$

$\mathrm{P}$ pressure. $\mathrm{N} \mathrm{m}^{-2}$

$r$ position of bubble, $m$

$r_{a} \quad$ position of bubble of bubble $a, \mathrm{~m}$

$r_{h} \quad$ position of bubble of bubble $b, \mathrm{~m}$

$R_{b} \quad$ radius of a bubble, $m$

$R e_{h} \quad$ Reynolds number for flow around a bubble, dimensionless

$t_{\mathrm{ab}} \quad$ time until collision between bubbles $a$ and b.s

u liquid velocity, $\mathrm{m} \mathrm{s}^{-1}$

$u_{\text {rise }} \quad$ rise velocity of a bubble, $\mathrm{ms}^{-1}$

$v \quad$ velocity of bubble, $\mathrm{m} \mathrm{s}^{-1}$

$V_{h} \quad$ volume of a bubble, $\mathrm{m}^{3}$
Greek letters

$\varepsilon_{l} \quad$ volume fraction liquid in computational cell. dimensionless

$\kappa \quad$ velocity gradient in calculating lift force, $s^{\cdots}$

$i_{l}$ bulk viscosity liquid, $\mathrm{kg} \mathrm{m}^{-1} \mathrm{~s}^{-1}$

$\mu_{t} \quad$ shear viscosity liquid, $\mathrm{kg} \mathrm{m}^{-1} \mathrm{~s}^{-1}$

$\rho_{g} \quad$ density gas phase, $\mathrm{kg} \mathrm{m}^{-3}$

$\rho_{l} \quad$ density liquid phase, $\mathrm{kg} \mathrm{m}^{-3}$

$\tau_{1} \quad$ stress tensor liquid, $\mathrm{N} \mathrm{m}^{-2}$

$\phi \quad$ typical Eulerian variable, dimensionless Ф momentum exchange gas to liquid. $\mathrm{N} \mathrm{m}$ $\Omega \quad$ vorticity in liquid phase, $s^{-1}$

\section{REFERENCES}

Auton, T. R. (1983) The dynamics of bubbles, drops and particles in motion in liquids. Ph.D. thesis, University of Cambridge, Cambridge, U.K.

Becker, S., Sokolichin, A. and Eigenberger, G. (1995) Gas-liquid flow in bubble columns and loop reactors. Part II. Comparison of detailed experiments and flow simulations. Chem. Engng Sci. 49, 5747.

Chen, J. J. J., Jamialahmadi, M. and Li, S. M. (1989) Effect of liquid depth on circulation in bubble columns: a visual study. Chem. Engng Res. Des. 67, 203.

Chesters, A. K., van Doorn, M. and Goossens, L. H. J. (1980) A general model for unconfined bubble plumes from extended sources. Int. J. Multiphase Flow 6, 499.

Clift, R., Grace, J. R. and Weber, M. E. (1978) Bubhles Drops, and Particle's. Academic Press. New York, U.S.A. 
Deckwer, W. D. and Schumpe, A. (1993) Improved tools for bubble column reactor design and scale up. Chem. Engng Sci. 48, 889.

Devanathan, N., Dudukovic, M. P., Lapin, A., Lübbert, A. (1995) Chaotic flow in bubble column reactors. Chem. Engng Sci. 50, 2661.

Duineveld, P. G. (1994) Bouncing and coalescence of two bubbles in water. Ph.D. thesis, Twente University, Enschede, The Netherlands.

Freedman, W. and Davidson, J. F. (1969) Holdup and liquid circulation in bubble columns. Trans. Instn Chem. Engng 47, T251.

Grienberger, J. and Hofman, H. (1992) Investigations and modelling of bubble columns. Chem. Engng $S c i$. 47, 2215.

Hjertager, B. H. and Morud, K. (1995) Computational fluid dynamics of bioreactors. Mod. Indent. Control. 16, 177.

Hoomans, B. P. B., Kuipers, J. A. M., Briels, W. J. and van Swaaij, W. P. M. (1996) Discrete particle simulation of bubble and slug formation in a two dimensional gas-fluidised bed: a hard sphere approach. Chem. Engng Sci. 51, 99.

Joshi, J. B. and Sharma, M. M. (1979) Some design features of radial baffles in sectionalised bubble columns. Can. J. Chem. Engng 57, 375.

Kuipers, J. A. M., van Duin, K. J., van Beckum, F. P. H. and van Swaaij, W. P. M. (1993) Computer simulation of the hydrodynamics of a two dimensional gas-fluidized bed. Comput. Chem. Engng 17, 839.

Lapin, A. and Lübbert, A. (1994) Numerical simulation of the dynamics of two phase gas-liquid flows in bubble columns. Chem. Engng Sci. 49, 3661.

Odar, F. and Hamilton, W. S. (1964) Forces on a sphere accelerating in a viscous liquid. J. Fluid Mech. 18, 302.

Ranade, V. V. (1992) Flow in bubble column: some numerical experiments. Chem. Engng Sci. 47, 1857.

Sokolichin, A. and Eigenberger, G. (1994) Gas-liquid flow in bubble columns and loop reactors. Part I. Detailed modelling and numerical simulation. Chem. Engng Sci. 49, 5735.

Svendsen, H. F., Jakobsen, H. A. and Torvik, R. (1992) Local flow structures in internal loop and bubble column reactors. Chem. Engng Sci. 47, 3297.

Torvik, R. and Svendsen, H. F. (1990) Modelling of slurry reactors, a fundamental approach. Chem. Engng Sci. 45, 2325.

Trapp, J. A. and Mortensen, G. A. (1993) A discrete particle model for bubble slug two phase flow. $J$. Comp. Phys. 107, 367.

Wijngaarden, L. van (1976) Some problems in the formulation of the equations for gas/liquid flows. In Theoretical and Applied Mechanics, ed. W. T. Koiter. North-Holland, Amsterdam, The Netherlands. 